Supporting Information

\title{
Enantioselective, Aerobic Copper-Catalyzed Intramolecular Carboamination and Carboetherification of Unactivated Alkenes
}

Tomasz Wdowik, Samuel L. Galster, Raul L. L. Carmo, and Sherry R. Chemler* Department of Chemistry

State University of New York at Buffalo, Buffalo, NY 14260, United States

schemler@buffalo.edu

Spectral Data of New Compounds 


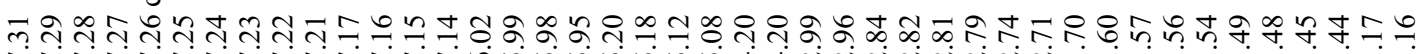

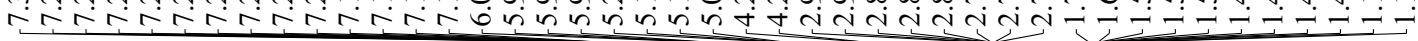
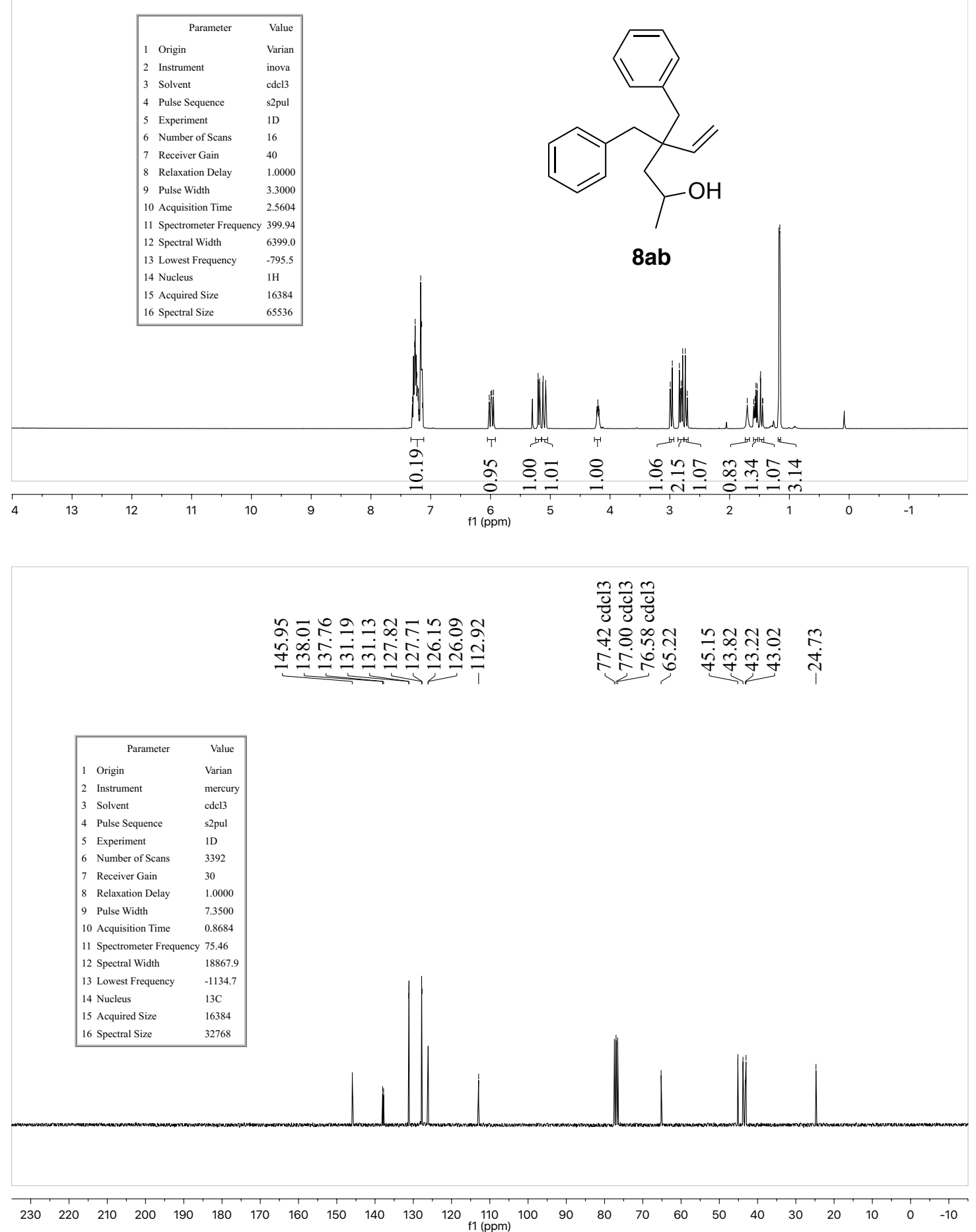

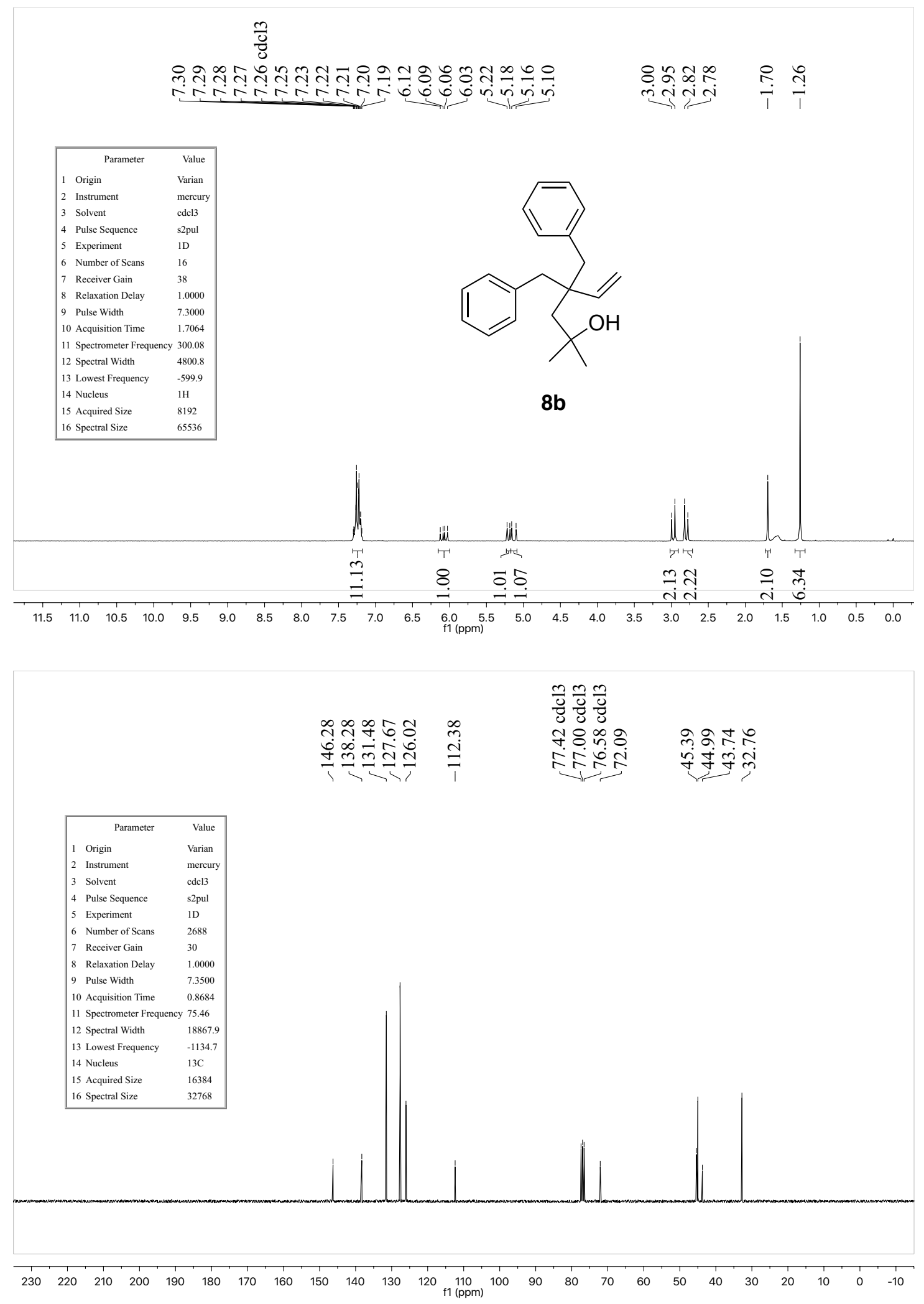

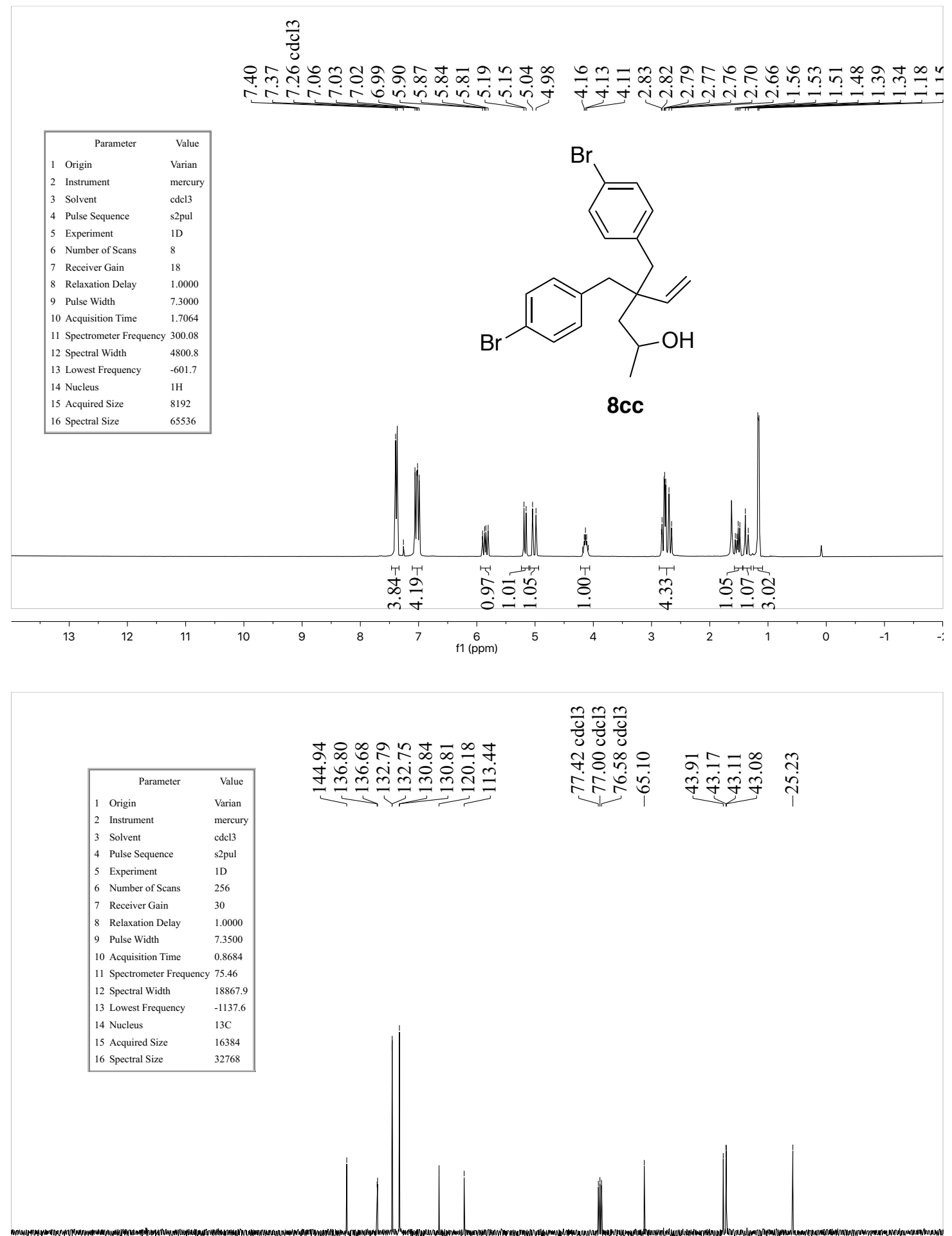

$\begin{array}{llllllllllllllllllllllllllll}230 & 220 & 210 & 200 & 190 & 180 & 170 & 160 & 150 & 140 & 130 & 120 & \begin{array}{c}110 \\ \mathrm{f} 1(\mathrm{ppm})\end{array} & 100 & 90 & 80 & 70 & 60 & 50 & 40 & 30 & 20 & 10 & 0 & -10\end{array}$ 
$\frac{m}{0}$

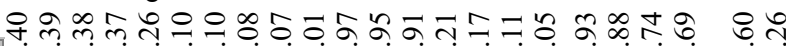

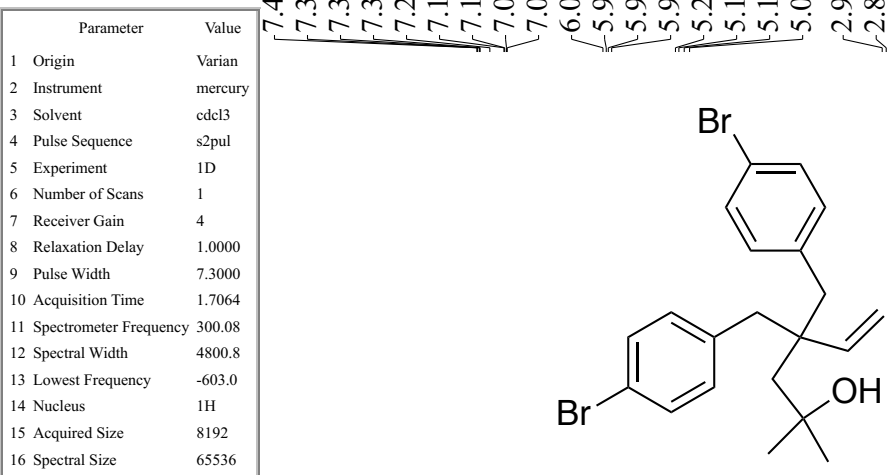

$8 c$
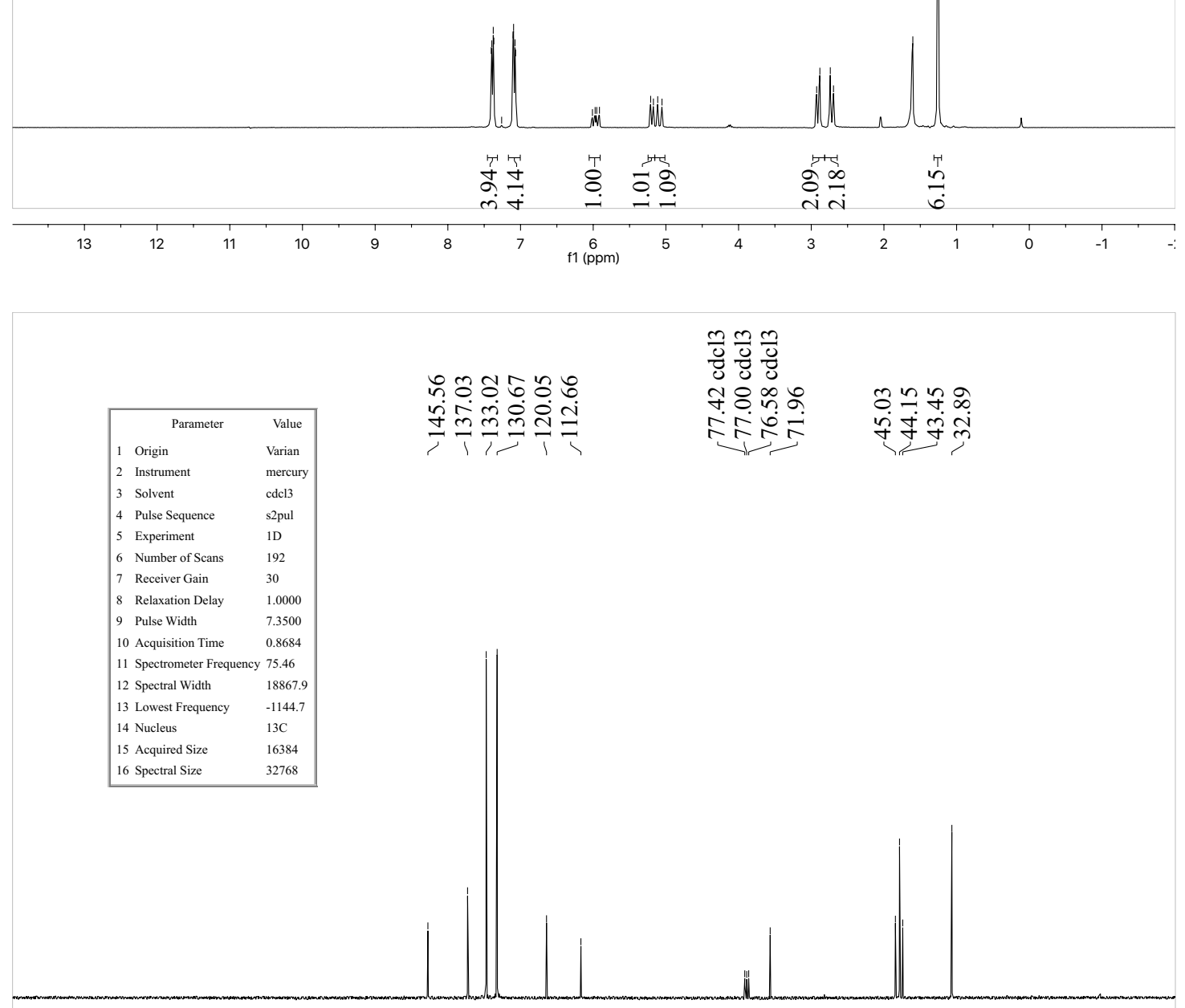

\begin{tabular}{lllllllllllllllllllllllllllllllllll}
\hline & 230 & 220 & 210 & 200 & 190 & 180 & 170 & 160 & 150 & 140 & 130 & 120 & 110 & 100 & 90 & 80 & 70 & 60 & 50 & 40 & 30 & 20 & 10 & 0 & -10
\end{tabular} 

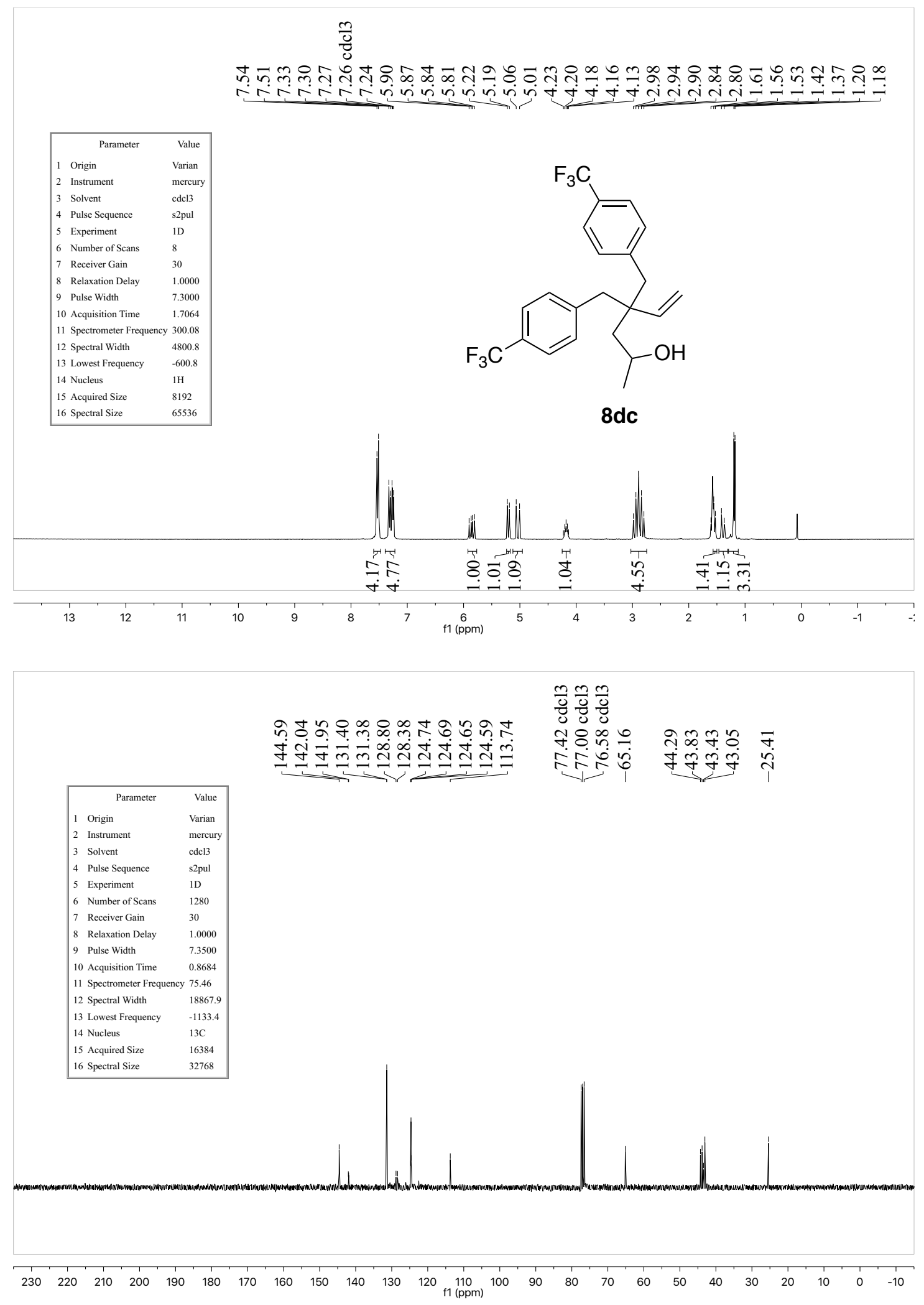

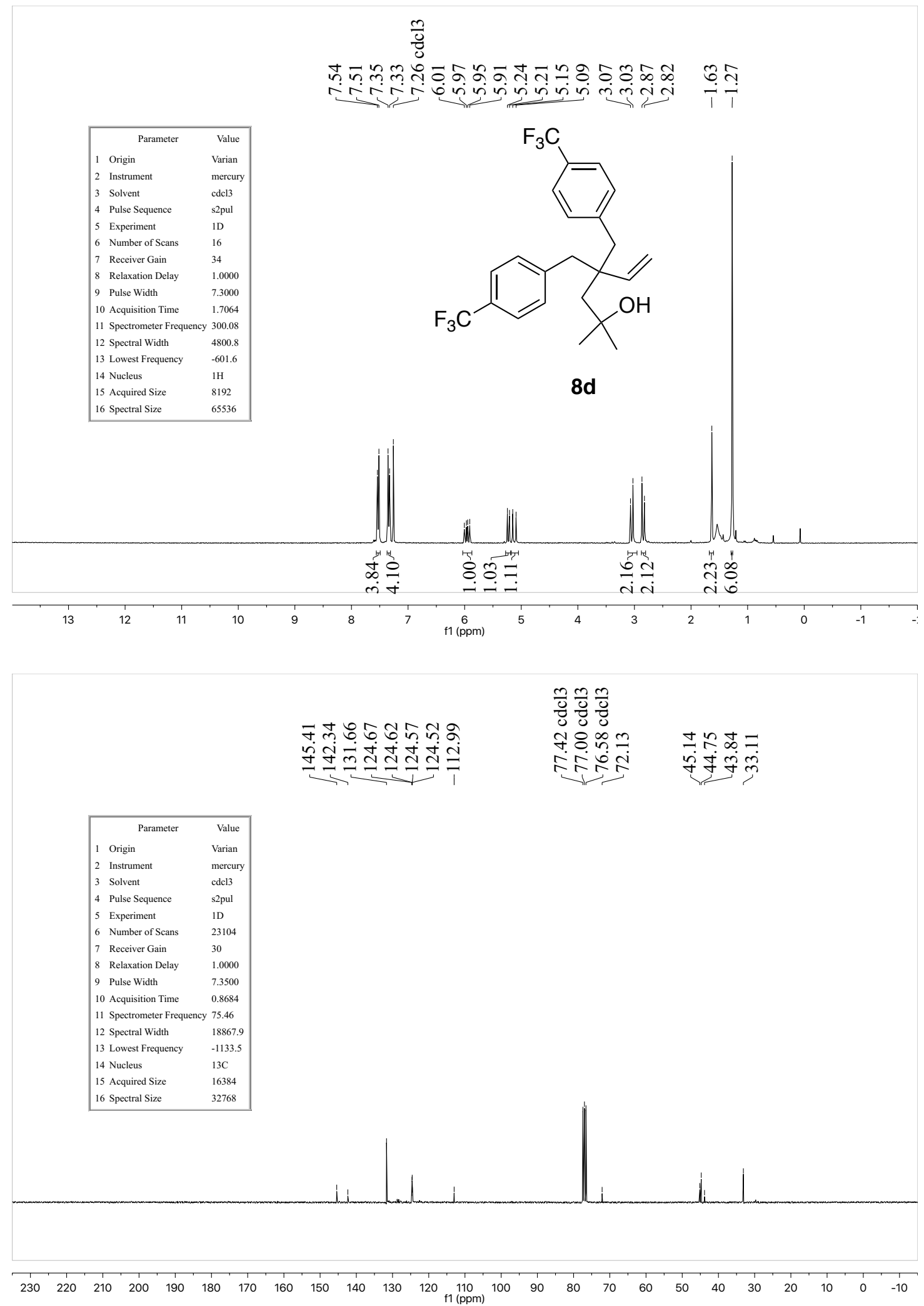


\begin{tabular}{|c|c|}
\hline Parameter & Value \\
\hline 1 Origin & Varian \\
\hline 2 Instrument & mercury \\
\hline 3 Solvent & cdc13 \\
\hline 4 Pulse Sequence & s2pul \\
\hline 5 Experiment & 1D \\
\hline 6 Number of Scans & 32 \\
\hline 7 Receiver Gain & 38 \\
\hline 8 Relaxation Delay & 1.0000 \\
\hline 9 Pulse Width & 7.3000 \\
\hline 10 Acquisition Time & 1.7064 \\
\hline \multicolumn{2}{|c|}{11 Spectrometer Frequency300.08 } \\
\hline 12 Spectral Width & 4800.8 \\
\hline 13 Lowest Frequency & -599.9 \\
\hline 14 Nucleus & $1 \mathrm{H}$ \\
\hline 15 Acquired Size & 8192 \\
\hline 16 Spectral Size & 65536 \\
\hline
\end{tabular}

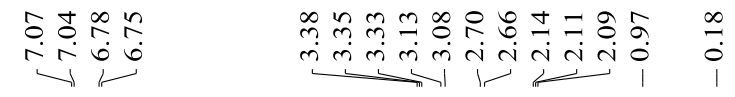

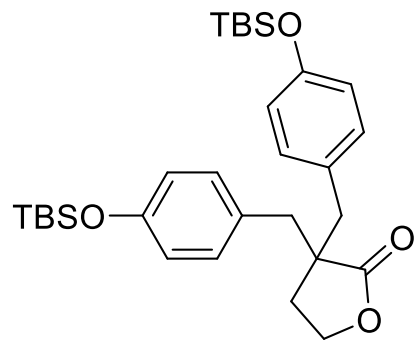

$8 e a$

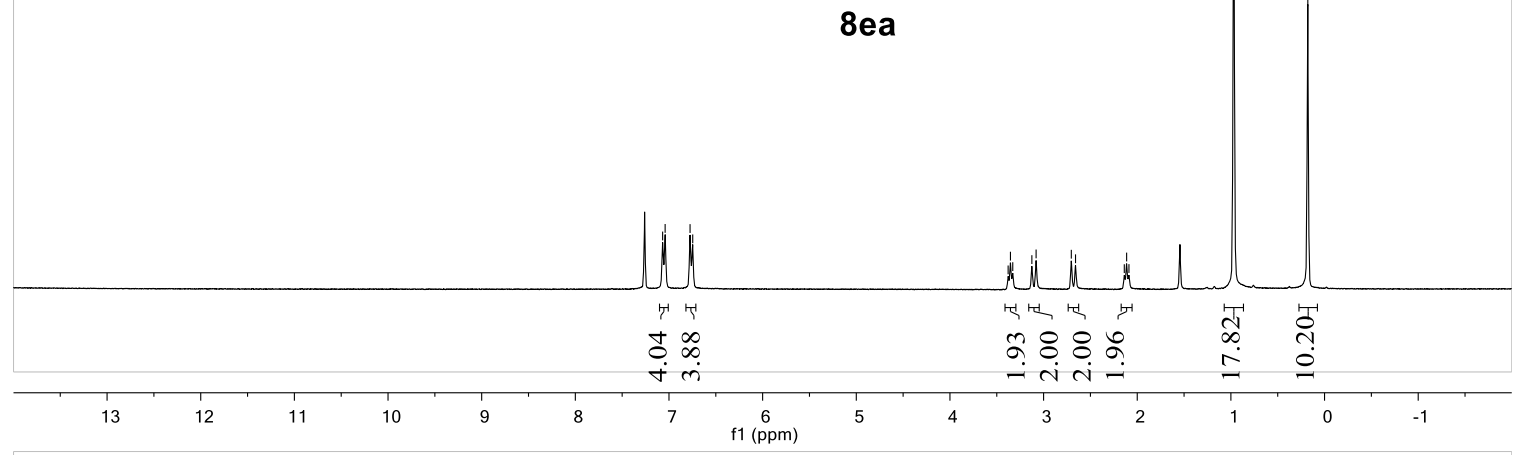

\begin{tabular}{|lll|}
\hline \multicolumn{1}{|c|}{ Parameter } & \multicolumn{1}{c|}{ Value } \\
1 Origin & Varian \\
2 & Instrument & mercury \\
3 & Solvent & cdcl3 \\
4 & Pulse Sequence & s2pul \\
5 & Experiment & $1 \mathrm{D}$ \\
6 & Number of Scans & 16128 \\
7 Receiver Gain & 30 \\
8 Relaxation Delay & 1.0000 \\
9 & Pulse Width & 7.3500 \\
10 Acquisition Time & 0.8684 \\
11 & Spectrometer Frequency75.46 \\
12 & Spectral Width & 18867.9 \\
13 Lowest Frequency & -1134.3 \\
14 Nucleus & $13 \mathrm{C}$ \\
15 Acquired Size & 16384 \\
16 Spectral Size & 32768 \\
\hline
\end{tabular}

$\frac{m}{0} \frac{m}{0} \frac{m}{0}$

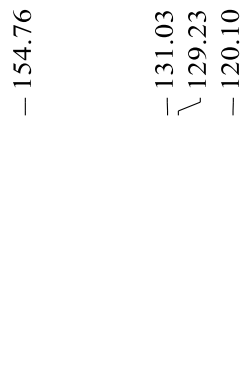

च

중ำ

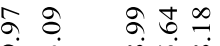

के $\stackrel{\infty}{\sim} \stackrel{\infty}{\sim}$

$\stackrel{\infty}{\circ}$




\begin{tabular}{|lll|}
\hline \multicolumn{1}{|c|}{ Parameter } & \multicolumn{1}{c|}{ Value } \\
1 & Origin & Varian \\
2 & Instrument & mercury \\
3 & Solvent & cdcl3 \\
4 & Pulse Sequence & s2pul \\
5 & Experiment & $1 \mathrm{D}$ \\
6 & Number of Scans & 32 \\
7 & Receiver Gain & 22 \\
8 8 & Relaxation Delay & 1.0000 \\
9 & Pulse Width & 7.3000 \\
10 Acquisition Time & 1.7064 \\
11 Spectrometer Frequency300.08 \\
12 Spectral Width & 4800.8 \\
13 Lowest Frequency & -599.3 \\
14 Nucleus & $1 \mathrm{H}$ \\
15 Acquired Size & 8192 \\
16 Spectral Size & 65536 \\
\hline
\end{tabular}

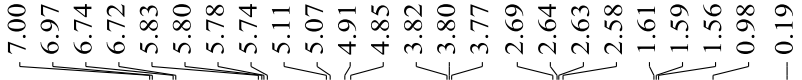

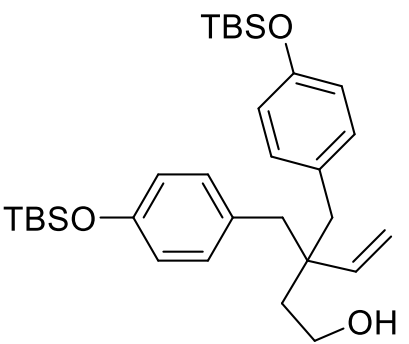

$8 e b$

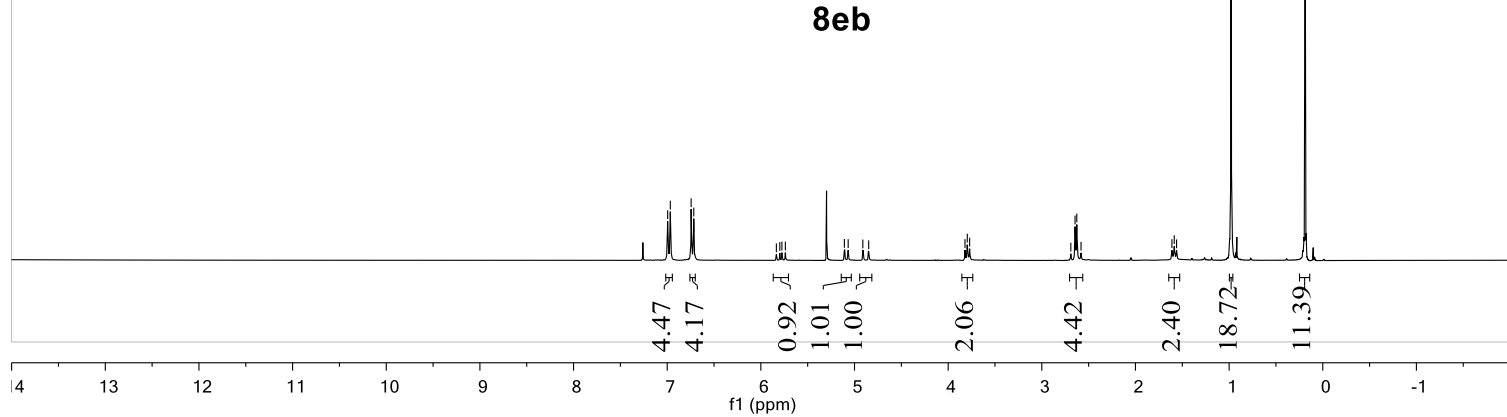

\begin{tabular}{|c|c|}
\hline Parameter & Value \\
\hline 1 Origin & Varian \\
\hline 2 Instrument & mercury \\
\hline 3 Solvent & cdcl3 \\
\hline 4 Pulse Sequence & s2pul \\
\hline 5 Experiment & 1D \\
\hline 6 Number of Scans & 3717 \\
\hline 7 Receiver Gain & 30 \\
\hline 8 Relaxation Delay & 1.0000 \\
\hline 9 Pulse Width & 7.3500 \\
\hline 10 Acquisition Time & 0.8684 \\
\hline \multicolumn{2}{|c|}{11 Spectrometer Frequency 75.46} \\
\hline 12 Spectral Width & 18867.9 \\
\hline 13 Lowest Frequency & -1134.1 \\
\hline 14 Nucleus & $13 \mathrm{C}$ \\
\hline 15 Acquired Size & 16384 \\
\hline 16 Spectral Size & 32768 \\
\hline
\end{tabular}

$\frac{m}{0} \frac{m}{0} \frac{m}{0}$

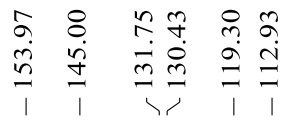

F.

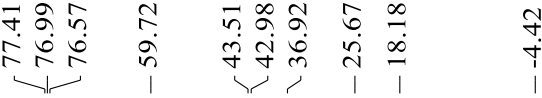

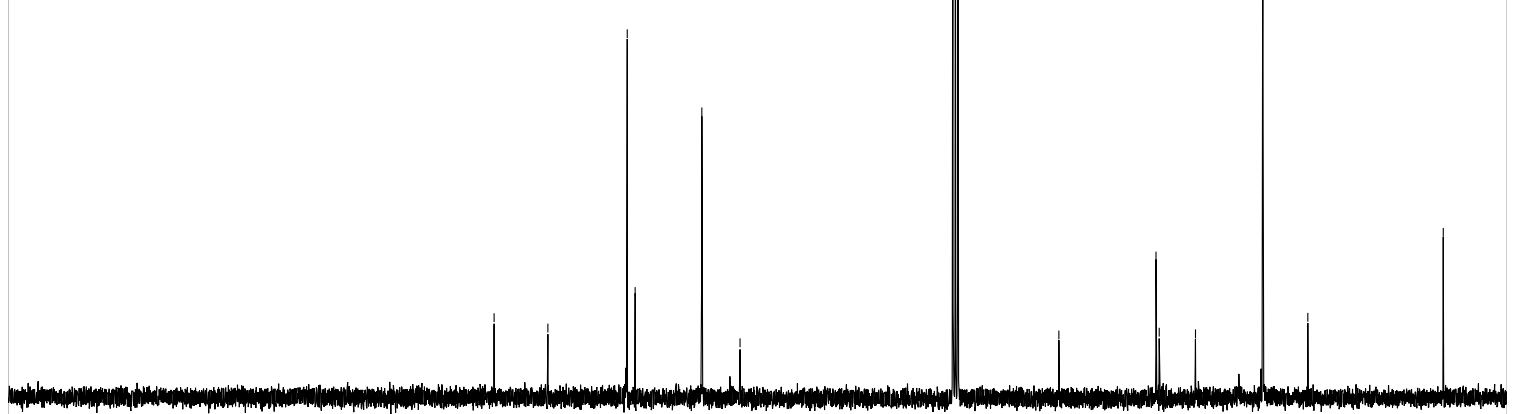

$\begin{array}{lllllllllllllllllllllllllllllllll}230 & 220 & 210 & 200 & 190 & 180 & 170 & 160 & 150 & 140 & 130 & 120 & \begin{array}{c}110 \\ \mathrm{f} 1(\mathrm{ppm})\end{array} & 100 & 90 & 80 & 70 & 60 & 50 & 40 & 30 & 20 & 10 & 0 & -10\end{array}$ 


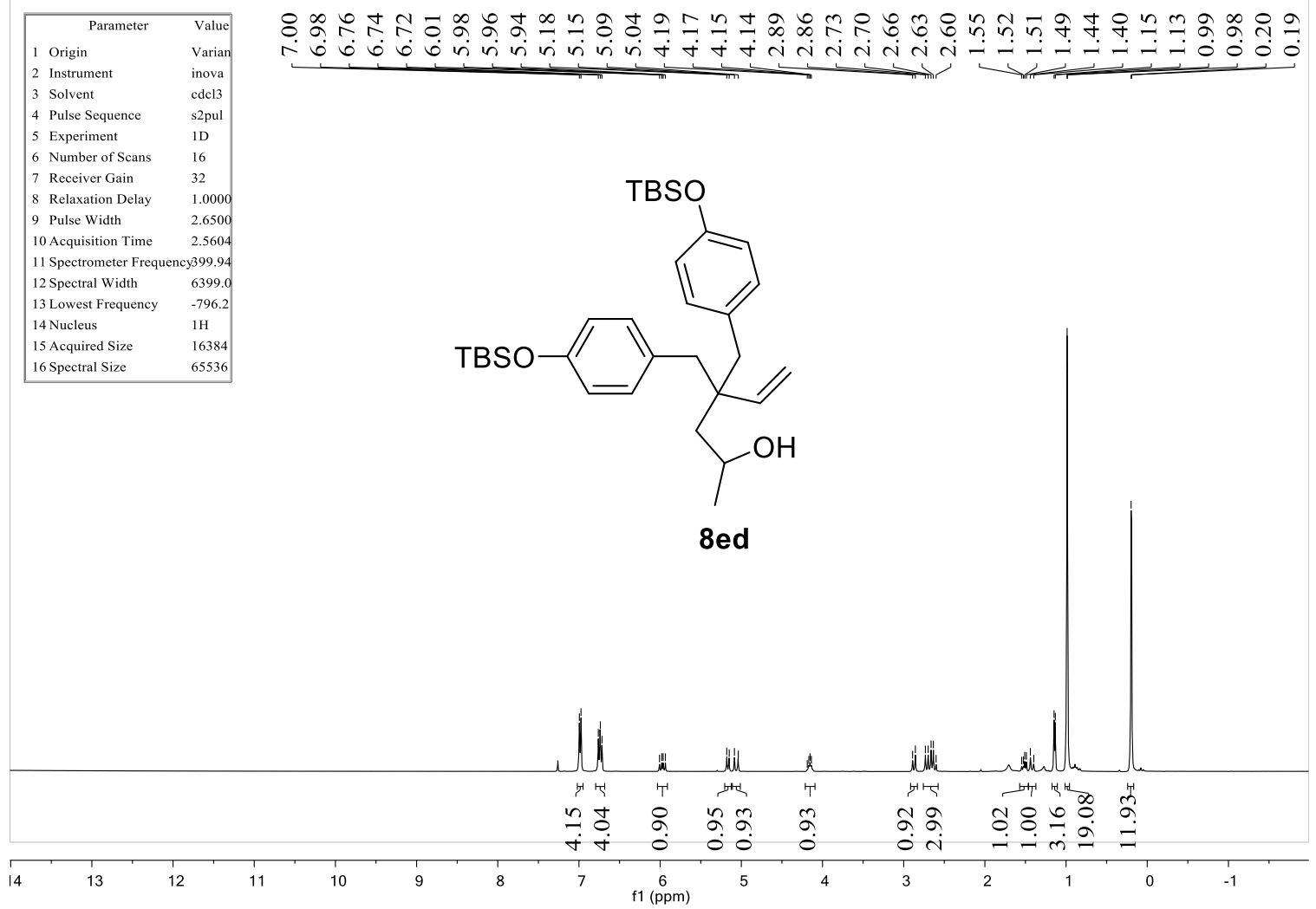

\begin{tabular}{|lll|}
\hline \multicolumn{1}{|c|}{ Parameter } & \multicolumn{1}{c|}{ Value } \\
1 & Origin & Varian \\
2 & Instrument & mercury \\
3 & Solvent & cdc13 \\
4 & Pulse Sequence & s2pul \\
5 & Experiment & $1 \mathrm{D}$ \\
6 & Number of Scans & 3560 \\
7 & Receiver Gain & 30 \\
8 & Relaxation Delay & 1.0000 \\
9 & Pulse Width & 7.3500 \\
10 Acquisition Time & 0.8684 \\
11 Spectrometer Frequency75.46 \\
12 Spectral Width & 18867.9 \\
13 Lowest Frequency & -1134.1 \\
14 Nucleus & $13 \mathrm{C}$ \\
15 Acquired Size & 16384 \\
16 Spectral Size & 32768 \\
\hline
\end{tabular}

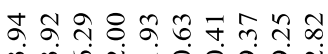

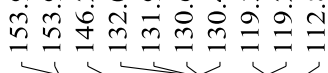

$\frac{m}{0} \frac{m}{0} \frac{m}{0}$

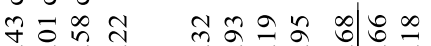

도용

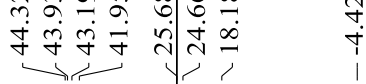

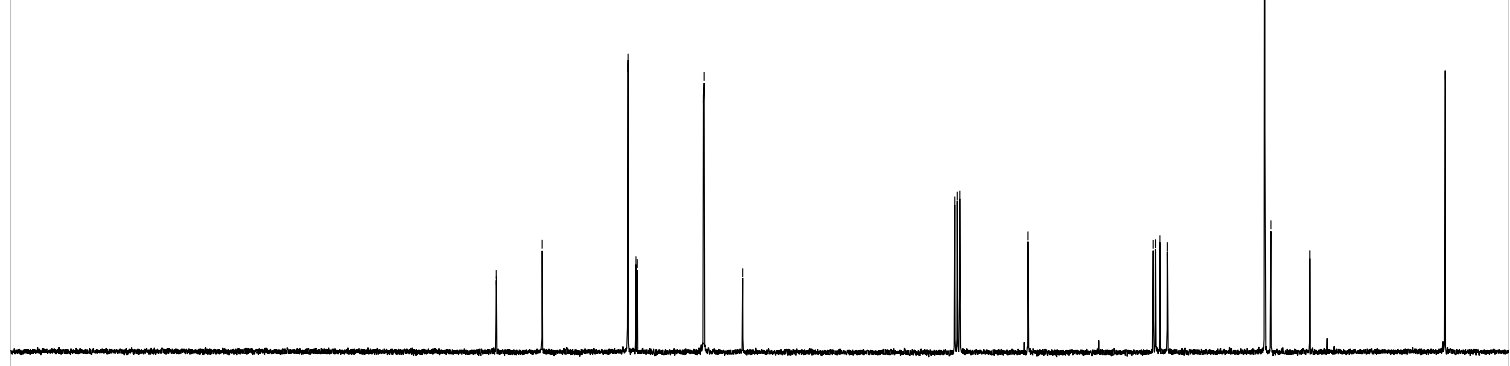

$\begin{array}{lllllllllllllllllllllllllll}230 & 220 & 210 & 200 & 190 & 180 & 170 & 160 & 150 & 140 & 130 & 120 & 110 & 100 & 90 & 80 & 70 & 60 & 50 & 40 & 30 & 20 & 10 & 0 & -10\end{array}$ 


\begin{tabular}{|c|c|}
\hline Parameter & Value \\
\hline 1 Origin & Varian \\
\hline 2 Instrument & mercury \\
\hline 3 Solvent & cdc13 \\
\hline 4 Pulse Sequence & s2pul \\
\hline 5 Experiment & 1D \\
\hline 6 Number of Scans & 32 \\
\hline 7 Receiver Gain & 22 \\
\hline 8 Relaxation Delay & 1.0000 \\
\hline 9 Pulse Width & 7.3000 \\
\hline 10 Acquisition Time & 1.7064 \\
\hline \multicolumn{2}{|c|}{11 Spectrometer Frequency300.08 } \\
\hline 12 Spectral Width & 4800.8 \\
\hline 13 Lowest Frequency & -599.9 \\
\hline 14 Nucleus & $1 \mathrm{H}$ \\
\hline 15 Acquired Size & 8192 \\
\hline 16 Spectral Size & 65536 \\
\hline
\end{tabular}

\section{$\frac{m}{d}$}

ำ ㅇำ 2 ㄷำ

N5

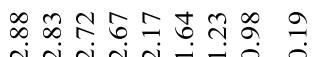

ing

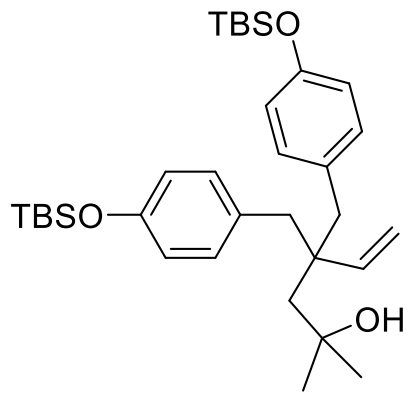

$8 \mathrm{e}$
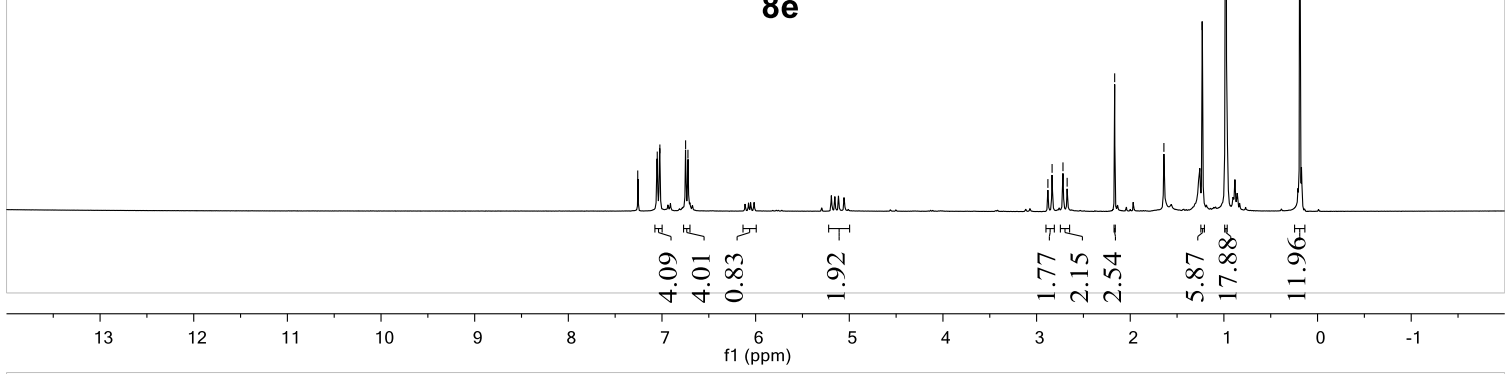

\begin{tabular}{|c|c|}
\hline Parameter & Value \\
\hline 1 Origin & Varian \\
\hline 2 Instrument & mercury \\
\hline 3 Solvent & cdel3 \\
\hline 4 Pulse Sequence & s2pul \\
\hline 5 Experiment & 1D \\
\hline 6 Number of Scans & 21997 \\
\hline 7 Receiver Gain & 30 \\
\hline 8 Relaxation Delay & 1.0000 \\
\hline 9 Pulse Width & 7.3500 \\
\hline 10 Acquisition Time & 0.8684 \\
\hline \multicolumn{2}{|c|}{11 Spectrometer Frequency 75.46} \\
\hline 12 Spectral Width & 18867.9 \\
\hline 13 Lowest Frequency & -1134.3 \\
\hline 14 Nucleus & $13 \mathrm{C}$ \\
\hline 15 Acquired Size & 16384 \\
\hline 16 Spectral Size & 32768 \\
\hline
\end{tabular}

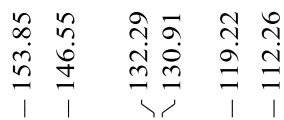

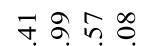

ㅇำ

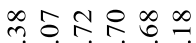

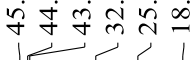

$\stackrel{\text { Tே}}{\stackrel{1}{+}}$

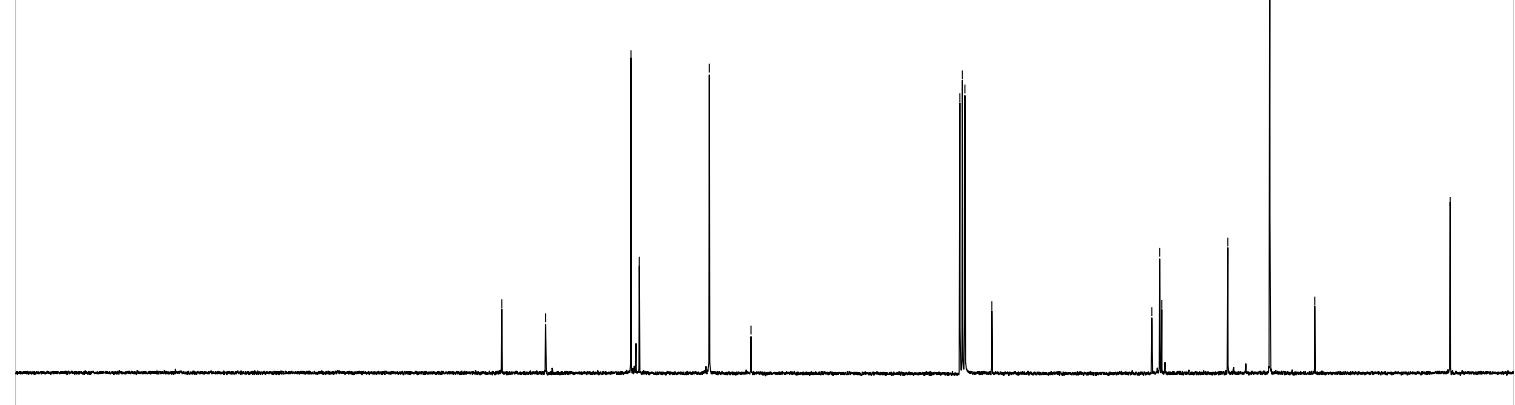

$\begin{array}{llllllllllllllllllllllllllllll} & 1 \\ 230 & 220 & 210 & 200 & 190 & 180 & 170 & 160 & 150 & 140 & 130 & 120 & 110 & 100 & 90 & 80 & 70 & 60 & 50 & 40 & 30 & 20 & 10 & 0 & -10\end{array}$ 
$\frac{m}{0}$

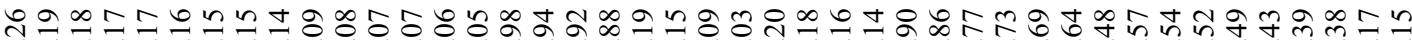

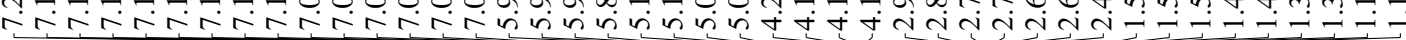

\begin{tabular}{|lll|}
\hline \multicolumn{1}{|c}{ Parameter } & \multicolumn{1}{c|}{ Value } \\
1 & Origin & Varian \\
2 & Instrument & mercury \\
3 & Solvent & cdc13 \\
4 & Pulse Sequence & s2pul \\
5 & Experiment & $1 \mathrm{D}$ \\
6 & Number of Scans & 8 \\
7 & Receiver Gain & 30 \\
8 & Relaxation Delay & 1.0000 \\
9 & Pulse Width & 7.3000 \\
10 & Acquisition Time & 1.7064 \\
11 & Spectrometer Frequency & 300.08 \\
12 & Spectral Width & 4800.8 \\
13 & Lowest Frequency & -599.6 \\
14 & Nucleus & $1 \mathrm{H}$ \\
15 & Acquired Size & 8192 \\
16 & Spectral Size & 65536 \\
\hline
\end{tabular}<smiles>C=CC(Cc1ccc(S(C)(C)C)cc1)(Cc1ccc(S(C)(=O)=O)cc1)CC(C)O</smiles>

16 Spectral Siz

$8 f c$
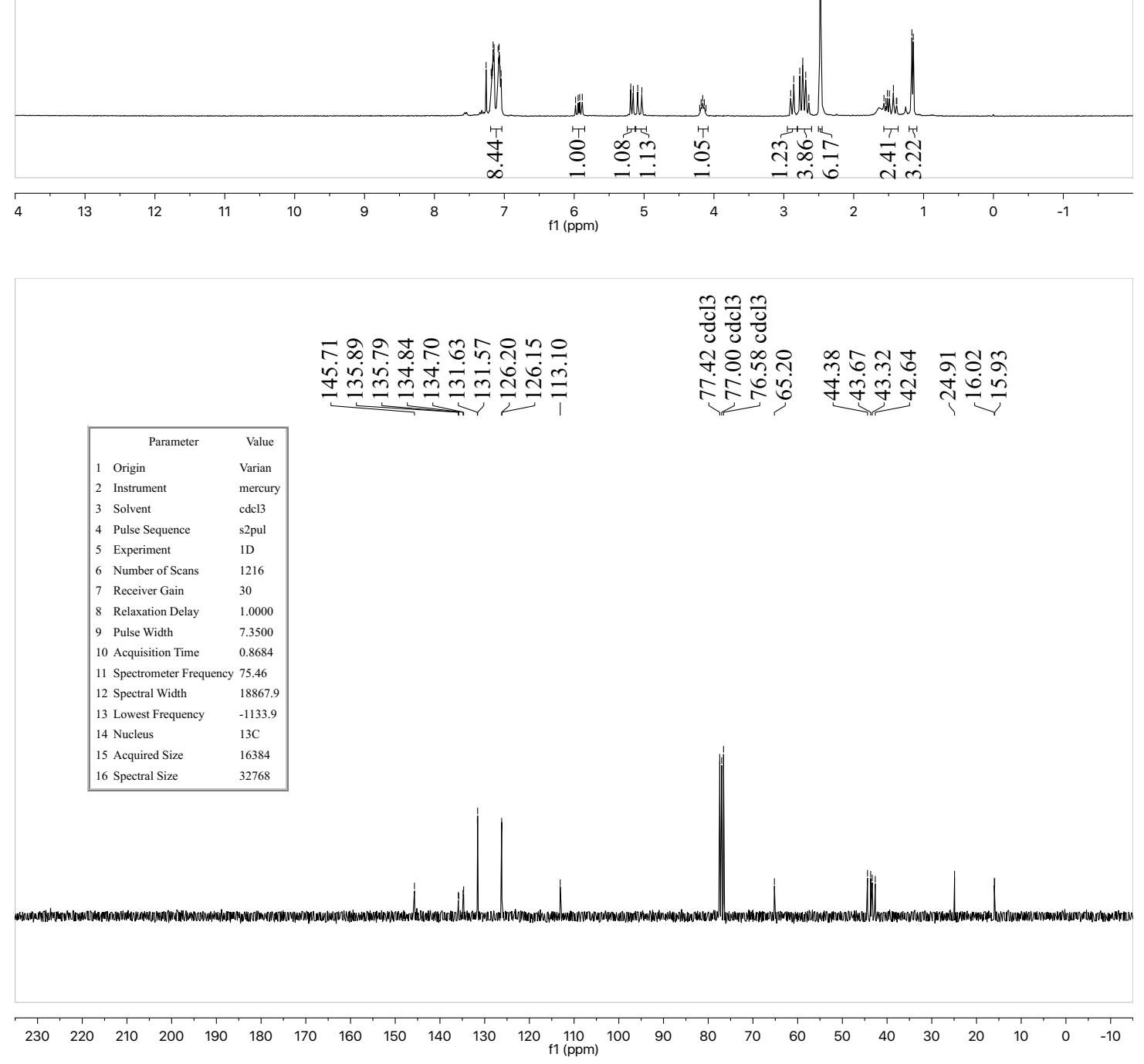

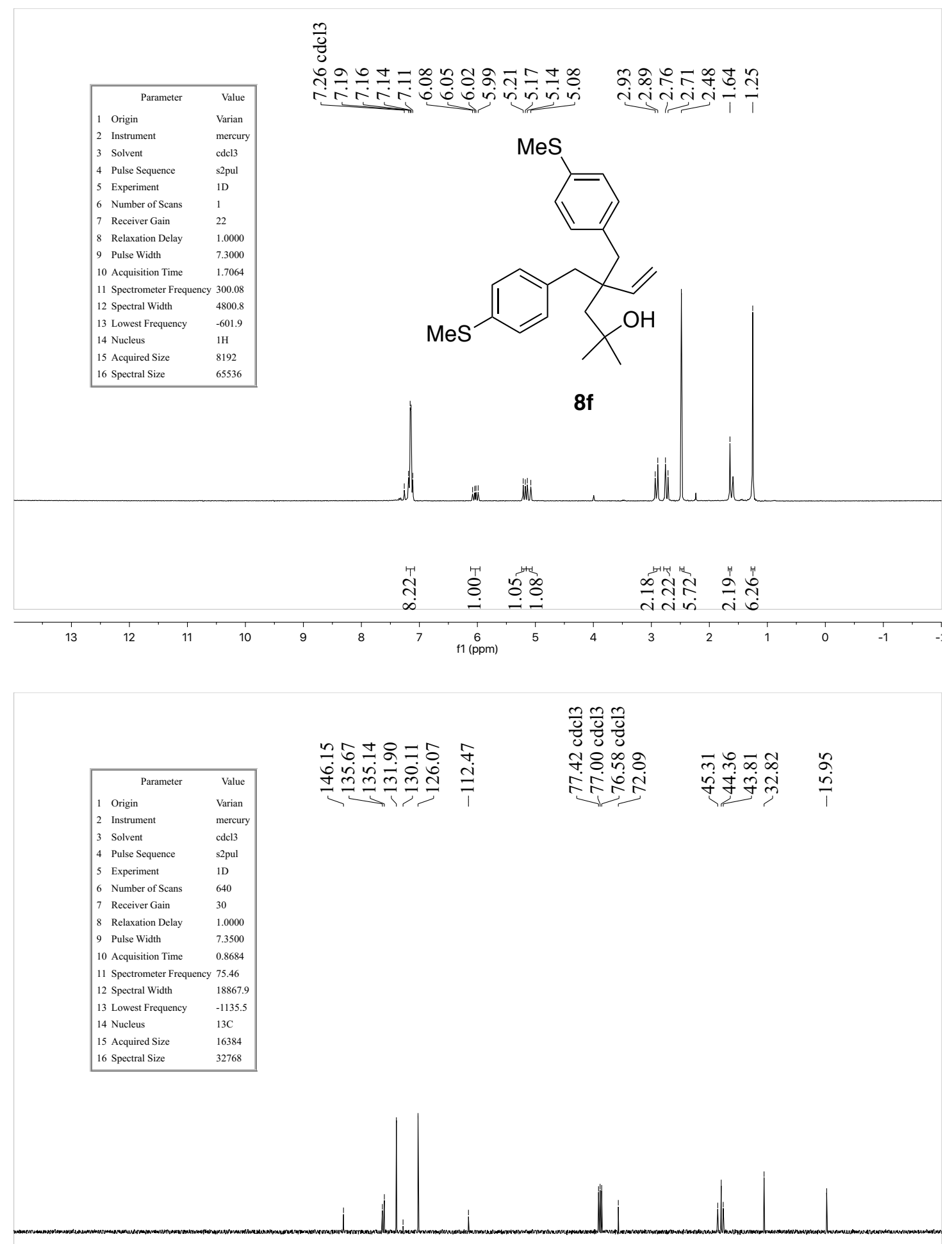

$\begin{array}{lllllllllllllllllllllllllll}230 & 220 & 210 & 200 & 190 & 180 & 170 & 160 & 150 & 140 & 130 & 120 & \begin{array}{c}110 \\ \mathrm{f} 1(\mathrm{ppm})\end{array} & 100 & 90 & 80 & 70 & 60 & 50 & 40 & 30 & 20 & 10 & 0 & -10\end{array}$ 


\begin{tabular}{|lll|}
\hline \multicolumn{1}{|c|}{ Parameter } & \multicolumn{1}{c|}{ Value } \\
1 & Origin & Varian \\
2 & Instrument & mercury \\
3 & Solvent & cdcl3 \\
4 & Pulse Sequence & s2pul \\
5 & Experiment & $1 \mathrm{D}$ \\
6 & Number of Scans & 16 \\
7 & Receiver Gain & 12 \\
8 & Relaxation Delay & 1.0000 \\
9 & Pulse Width & 7.3000 \\
10 Acquisition Time & 1.7064 \\
11 Spectrometer Frequency300.08 \\
12 Spectral Width & 4800.8 \\
13 Lowest Frequency & -599.8 \\
14 Nucleus & $1 \mathrm{H}$ \\
15 Acquired Size & 8192 \\
16 Spectral Size & 65536 \\
\hline
\end{tabular}

旁

œ م

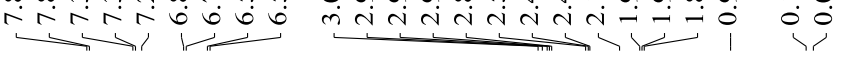<smiles>CC(C)(C)Oc1ccc(CC2(Cc3ccc(OC(C)(C)C)cc3)CCN([PH3])C2=O)cc1</smiles>

$6 f a$

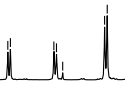

सम

ڤूํำ के

Ts
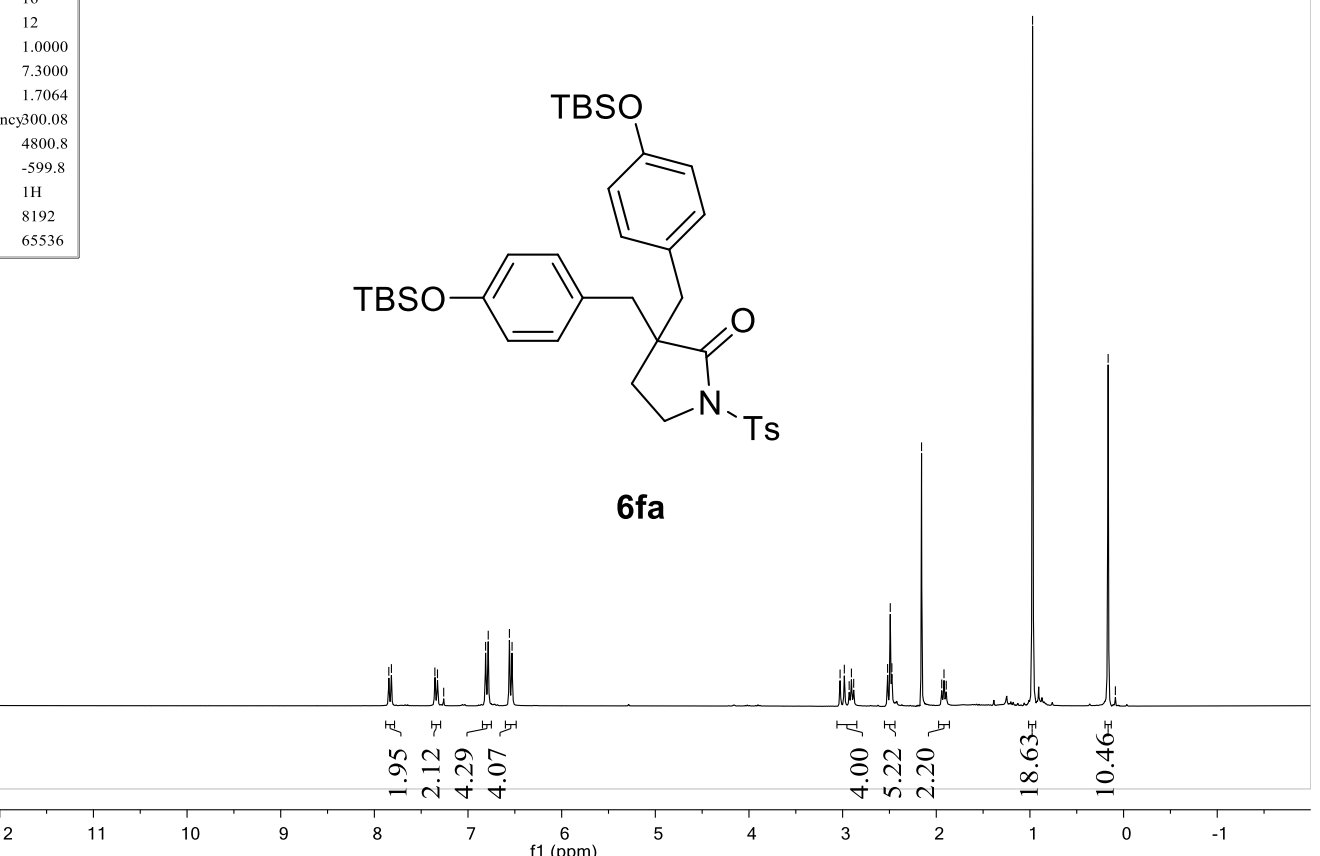

\begin{tabular}{|lll|}
\hline \multicolumn{1}{|c}{ Parameter } & \multicolumn{1}{c}{ Value } \\
1 1 & Origin & Varian \\
2 & Instrument & mercury \\
3 & Solvent & cdcl3 \\
4 & Pulse Sequence & s2pul \\
5 & Experiment & $1 \mathrm{D}$ \\
6 & Number of Scans & 25456 \\
7 & Receiver Gain & 30 \\
8 & Relaxation Delay & 1.0000 \\
9 & Pulse Width & 7.3500 \\
10 Acquisition Time & 0.8684 \\
11 Spectrometer Frequency75.46 \\
12 Spectral Width & 18867.9 \\
13 Lowest Frequency & -1136.6 \\
14 Nucleus & $13 \mathrm{C}$ \\
15 Acquired Size & 16384 \\
16 Spectral Size & 32768 \\
\hline
\end{tabular}

$\frac{m}{0} \frac{m}{0} \frac{m}{0}$

y 2 in

กิน

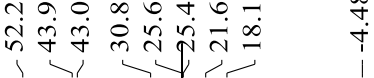

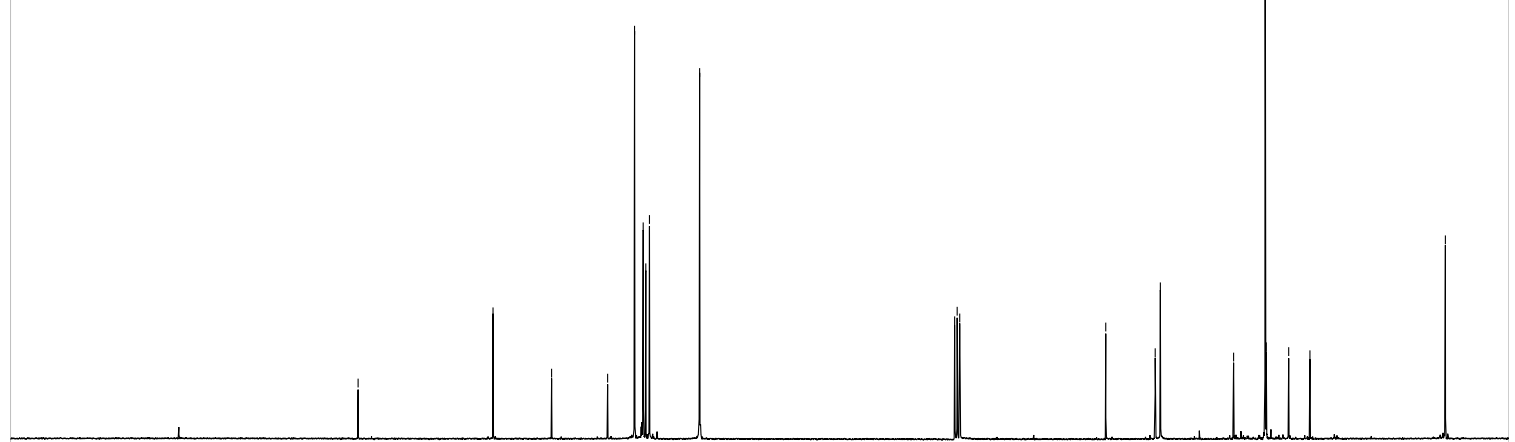

$\begin{array}{lllllllllllllllllllllllllll}230 & 220 & 210 & 200 & 190 & 180 & 170 & 160 & 150 & 140 & 130 & 120 & \begin{array}{l}110 \\ 110\end{array} & 100 & 90 & 80 & 70 & 60 & 50 & 40 & 30 & 20 & 10 & 0 & -10\end{array}$ 


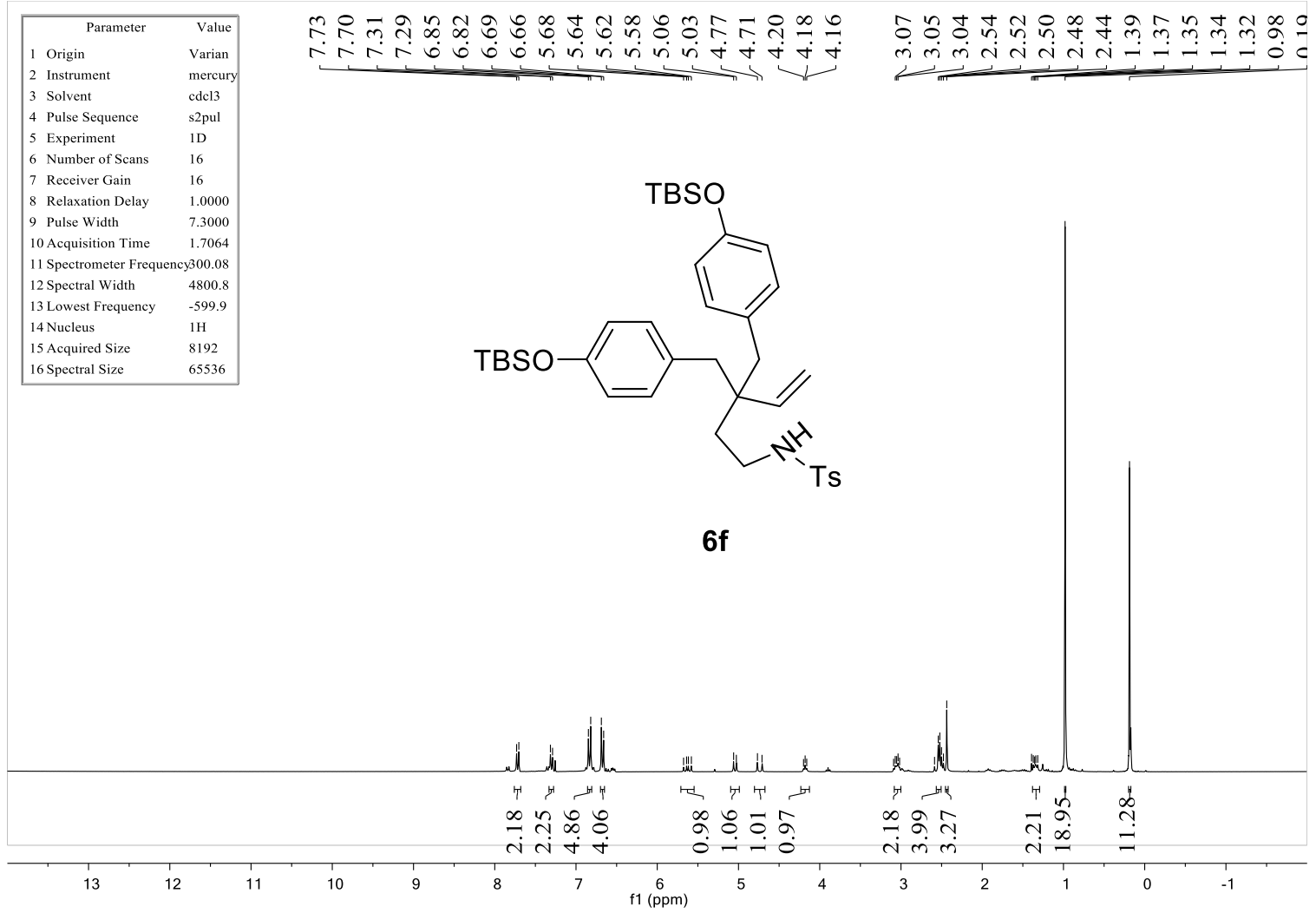

\begin{tabular}{|lll|}
\hline \multicolumn{1}{|c|}{ Parameter } & \multicolumn{1}{c|}{ Value } \\
1 & Origin & Varian \\
2 & Instrument & mercury \\
3 & Solvent & cdc13 \\
4 & Pulse Sequence & s2pul \\
5 & Experiment & $1 \mathrm{D}$ \\
6 & Number of Scans & 1060 \\
7 & Receiver Gain & 30 \\
8 & Relaxation Delay & 1.0000 \\
9 & Pulse Width & 7.3500 \\
10 Acquisition Time & 0.8684 \\
11 Spectrometer Frequency75.46 \\
12 Spectral Width & 18867.9 \\
13 Lowest Frequency & -1134.1 \\
14 Nucleus & $13 \mathrm{C}$ \\
15 Acquired Size & 16384 \\
16 Spectral Size & 32768 \\
\hline
\end{tabular}

पํํำ

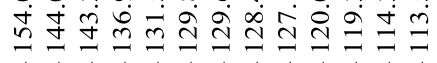

$\frac{m}{g} \frac{m}{0} \frac{m}{g}$

\%

손

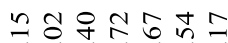

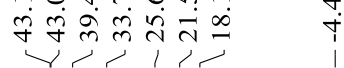

$\underset{\forall}{\dot{T}}$

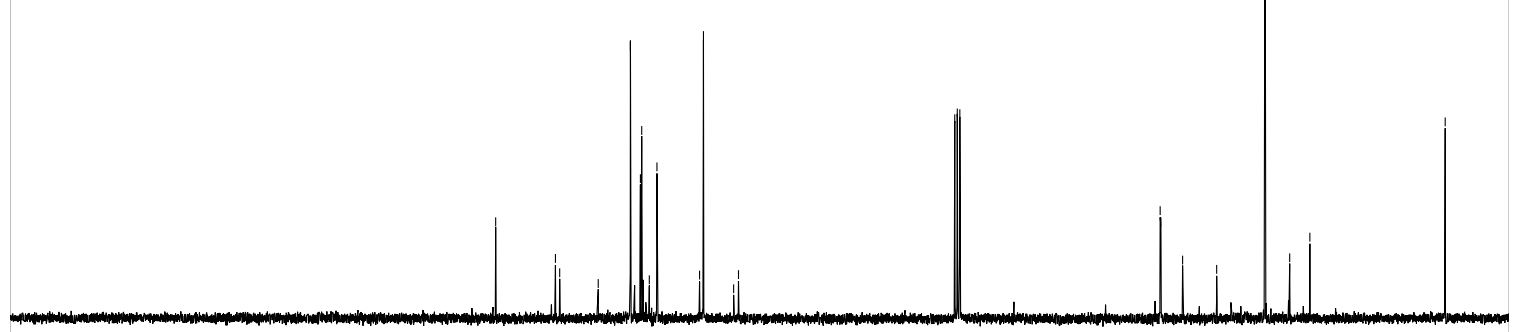

$\begin{array}{lllllllllllllllllllllllll}230 & 220 & 210 & 200 & 190 & 180 & 170 & 160 & 150 & 140 & 130 & 120 & \begin{array}{c}110 \\ 110\end{array} & 100 & 90 & 80 & 70 & 60 & 50 & 40 & 30 & 20 & 10 & 0 & -10\end{array}$ 
$\frac{m}{0}$

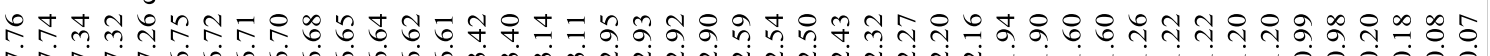

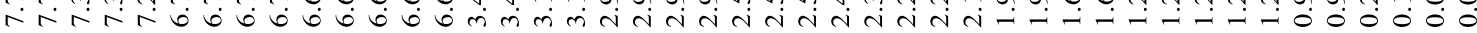

\begin{tabular}{|lll|}
\hline \multicolumn{1}{|c|}{ Parameter } & \multicolumn{1}{c|}{ Value } \\
1 & Origin & Varian \\
2 & Instrument & mercury \\
3 & Solvent & cdcl3 \\
4 & Pulse Sequence & s2pul \\
5 & Experiment & $1 \mathrm{D}$ \\
6 & Number of Scans & 16 \\
7 & Receiver Gain & 22 \\
8 & Relaxation Delay & 1.0000 \\
9 & Pulse Width & 7.3000 \\
10 Acquisition Time & 1.7064 \\
11 Spectrometer Frequency300.08 \\
12 Spectral Width & 4800.8 \\
13 Lowest Frequency & -599.8 \\
14 Nucleus & $1 \mathrm{H}$ \\
15 Acquired Size & 8192 \\
16 Spectral Size & 65536 \\
\hline
\end{tabular}

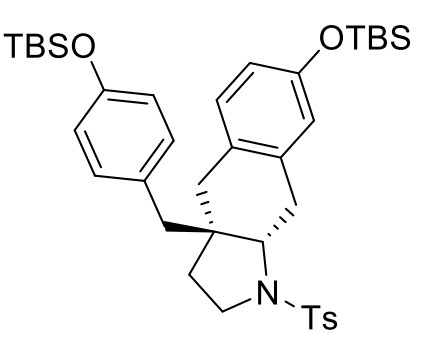

$7 f$

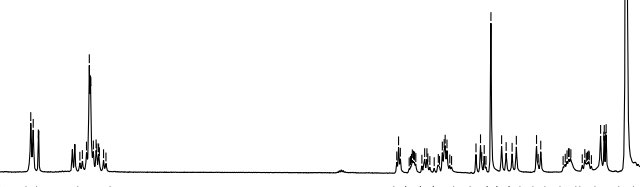

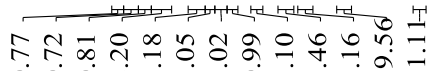

स्म

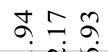

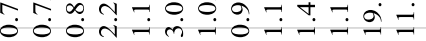

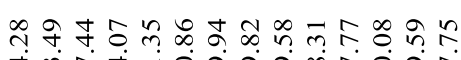

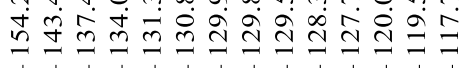

$\frac{m}{0} \frac{m}{0} \frac{m}{0}$

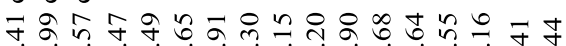

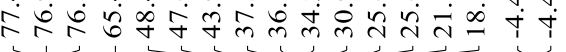

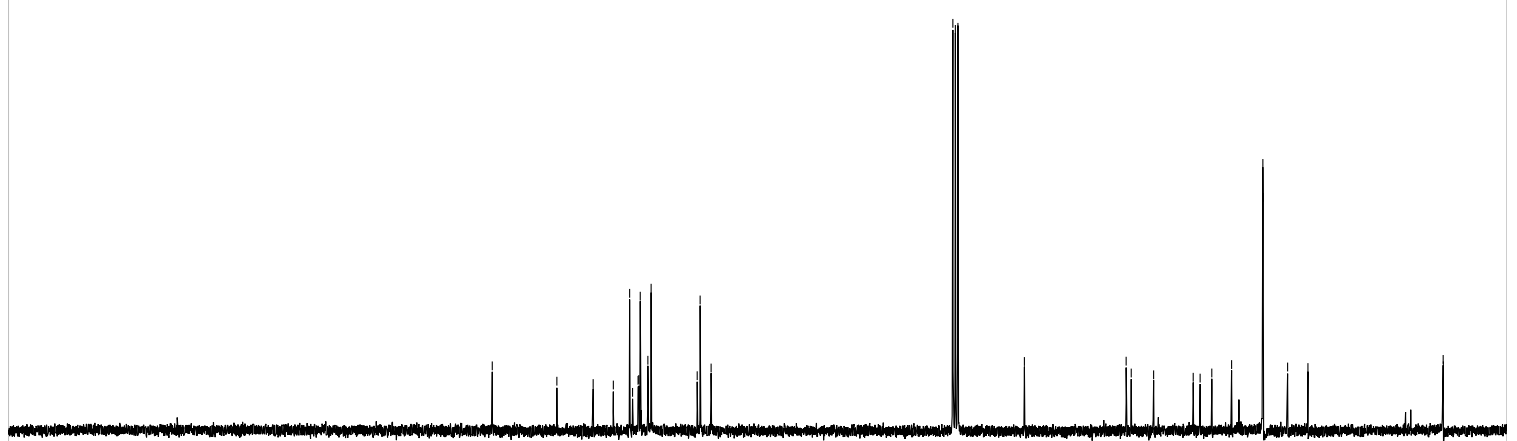

$\begin{array}{lllllllllllllllllllllllllllllll}230 & 220 & 210 & 200 & 190 & 180 & 170 & 160 & 150 & 140 & 130 & 120 & 110 & 100 & 90 & 80 & 70 & 60 & 50 & 40 & 30 & 20 & 10 & 0 & -10\end{array}$ 


\begin{tabular}{|lll|}
\hline & \multicolumn{1}{|c|}{ Parameter } & \multicolumn{1}{c|}{ Value } \\
1 & Origin & Varian \\
2 & Instrument & mercury \\
3 & Solvent & cdcl3 \\
4 & Pulse Sequence & s2pul \\
5 & Experiment & $1 \mathrm{D}$ \\
6 & Number of Scans & 16 \\
7 & Receiver Gain & 10 \\
8 & Relaxation Delay & 1.0000 \\
9 & Pulse Width & 7.3000 \\
10 & Acquisition Time & 1.7064 \\
11 & Spectrometer Frequency & 300.08 \\
12 & Spectral Width & 4800.8 \\
13 & Lowest Frequency & -599.9 \\
14 & Nucleus & $1 \mathrm{H}$ \\
15 & Acquired Size & 8192 \\
16 & Spectral Size & 65536 \\
\hline
\end{tabular}

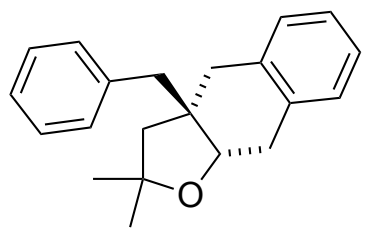

$9 b$
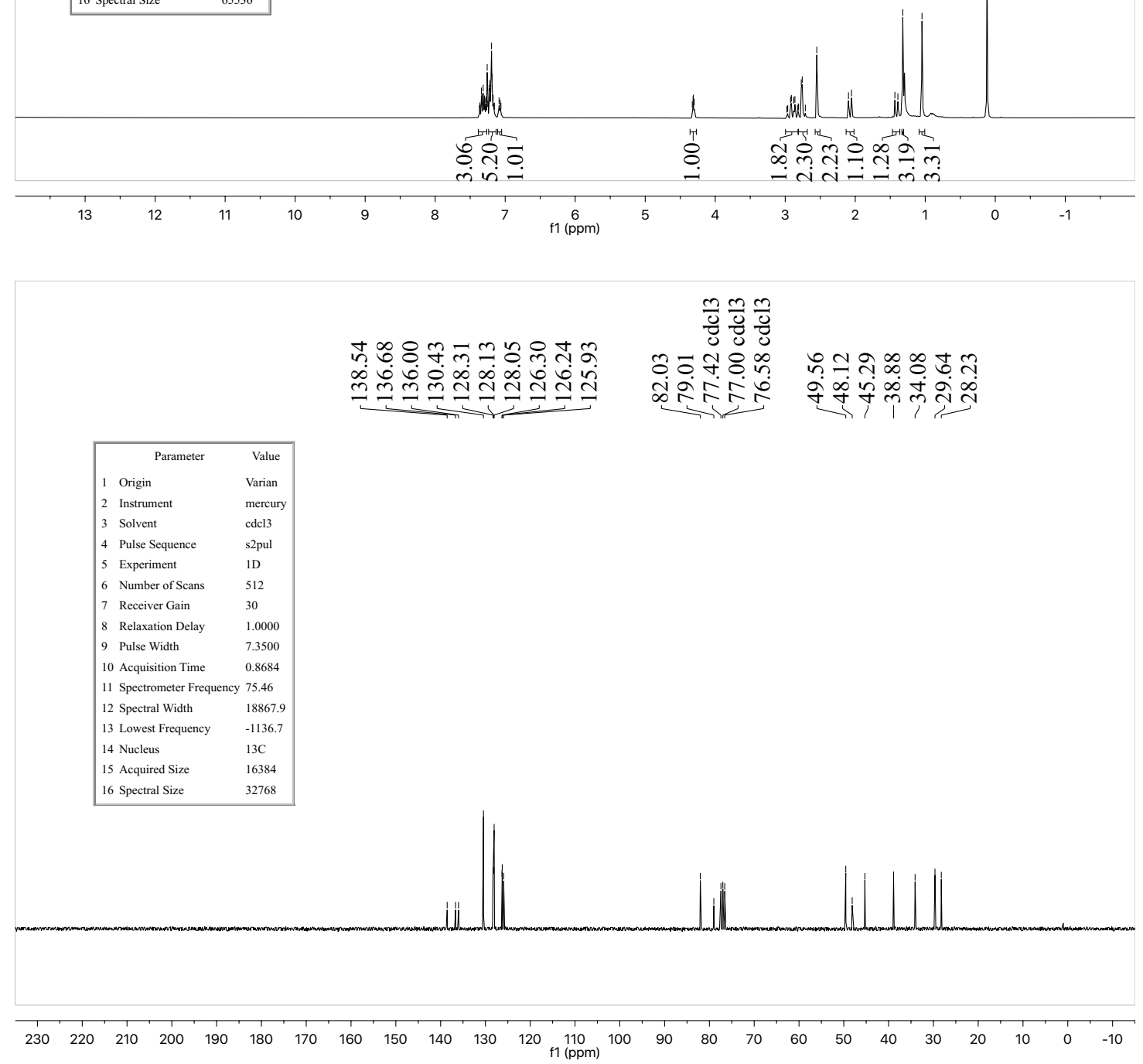

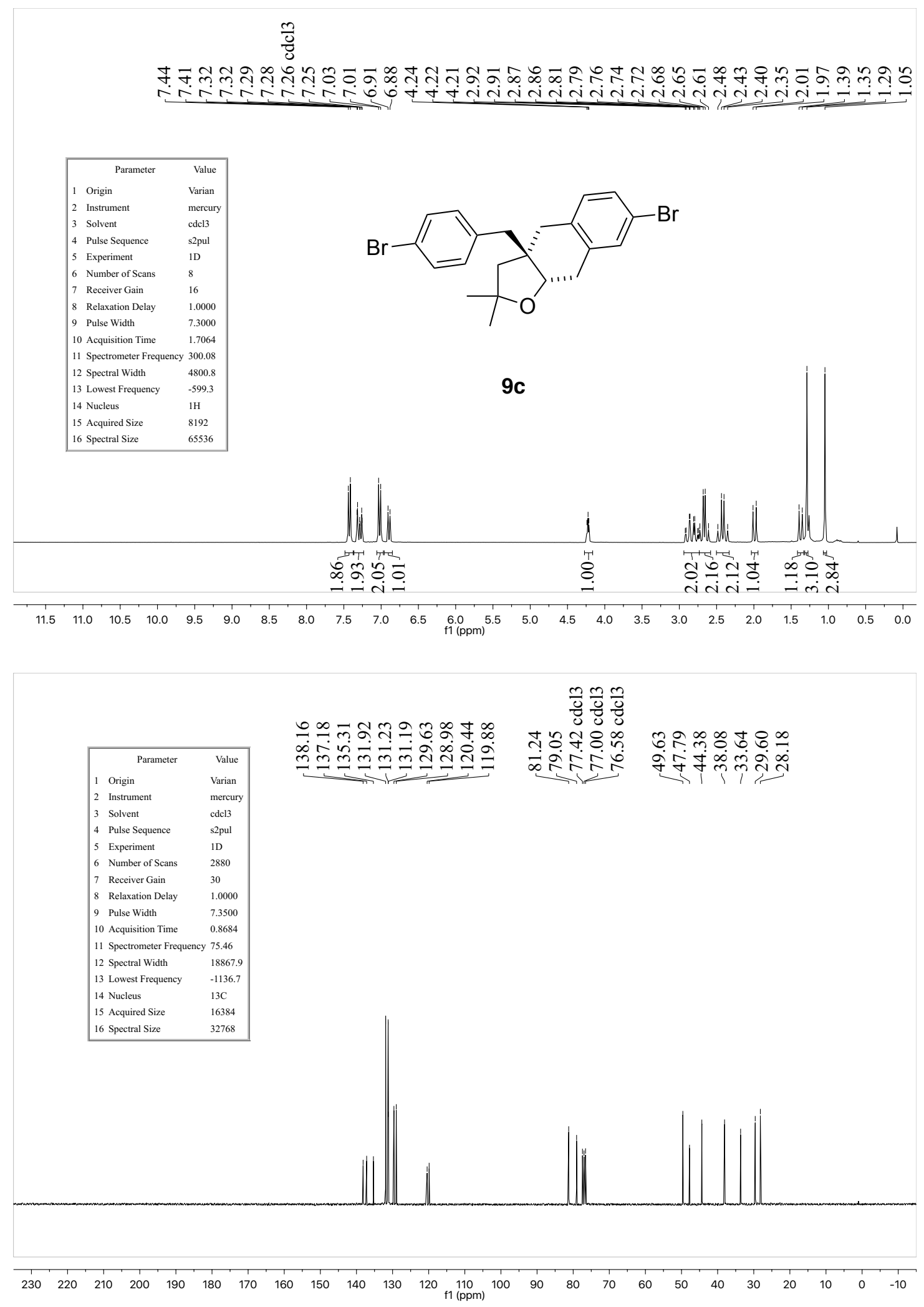

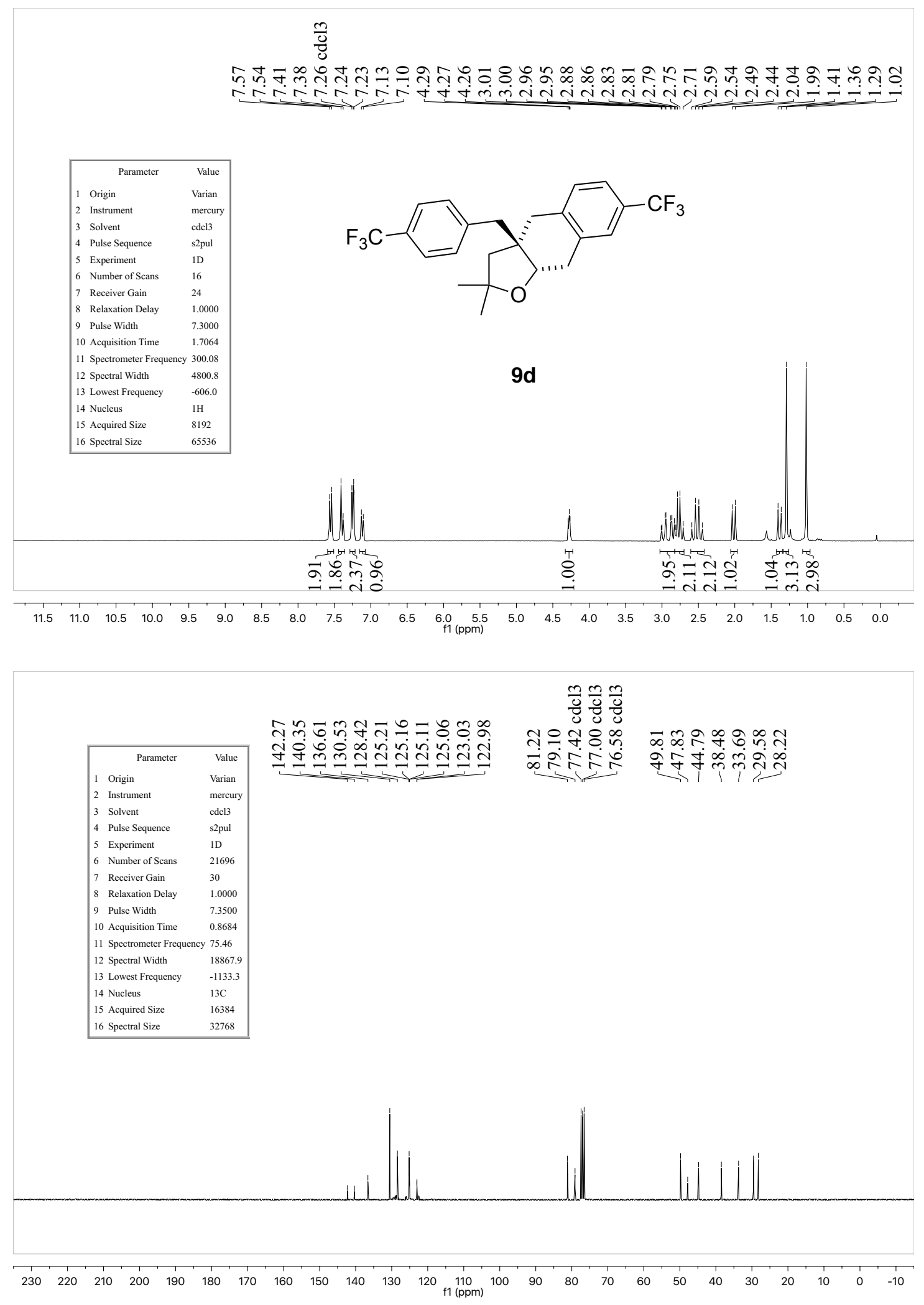


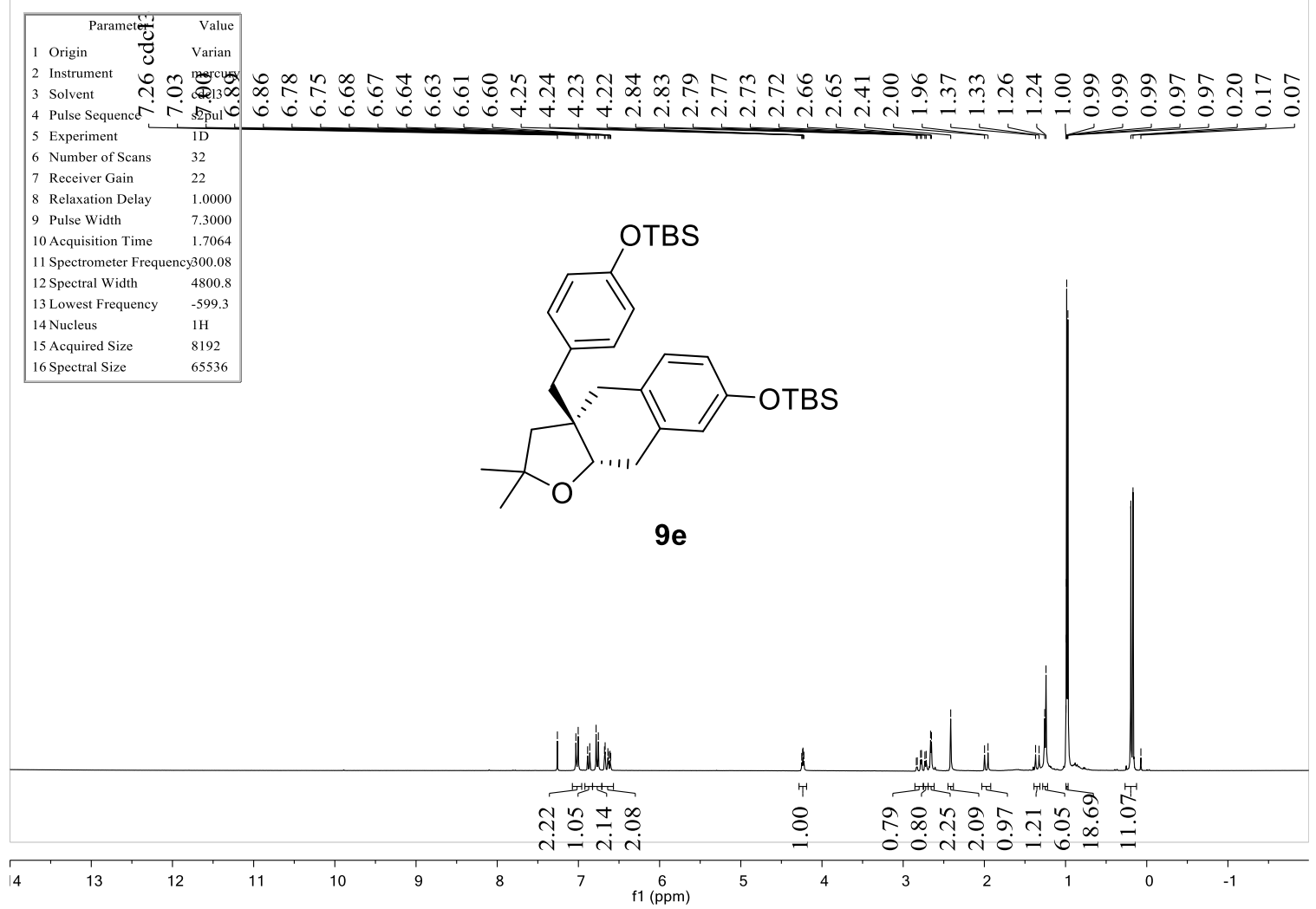

\begin{tabular}{|lll|}
\hline \multicolumn{1}{|c|}{ Parameter } & \multicolumn{1}{c|}{ Value } \\
1 & Origin & Varian \\
2 & Instrument & mercury \\
3 & Solvent & cdc13 \\
4 & Pulse Sequence & s2pul \\
5 & Experiment & $1 \mathrm{D}$ \\
6 & Number of Scans & 23040 \\
7 & Receiver Gain & 30 \\
8 & Relaxation Delay & 1.0000 \\
9 & Pulse Width & 7.3500 \\
10 Acquisition Time & 0.8684 \\
11 Spectrometer Frequency75.46 \\
12 Spectral Width & 18867.9 \\
13 Lowest Frequency & -1134.3 \\
14 Nucleus & $13 \mathrm{C}$ \\
15 Acquired Size & 16384 \\
16 Spectral Size & 32768 \\
\hline
\end{tabular}

$\frac{m}{\bar{s}} \frac{m}{\tilde{s}} \frac{m}{\delta}$

우유ำ

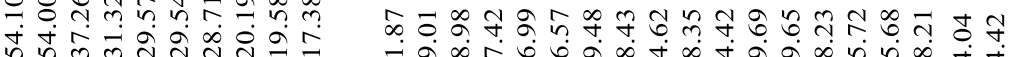

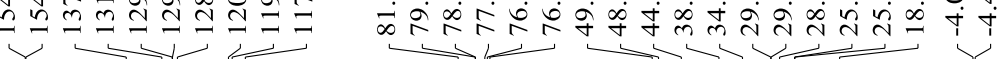

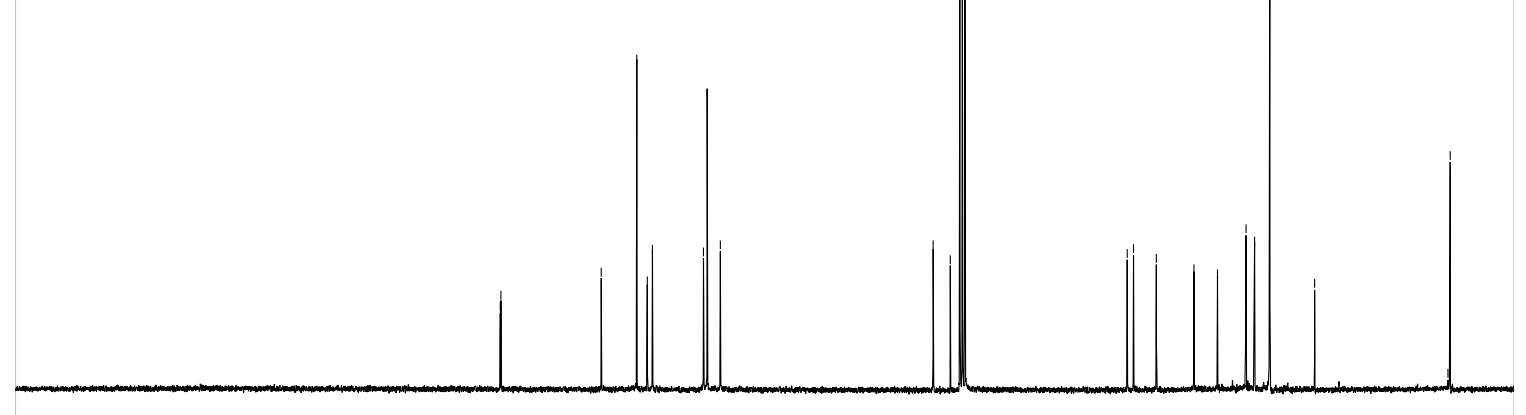

$\begin{array}{llllllllllllllllllllllllll}230 & 220 & 210 & 200 & 190 & 180 & 170 & 160 & 150 & 140 & 130 & 120 & \begin{array}{c}110 \\ \mathrm{f} 1(\mathrm{ppm})\end{array} & 100 & 90 & 80 & 70 & 60 & 50 & 40 & 30 & 20 & 10 & 0 & -10\end{array}$ 


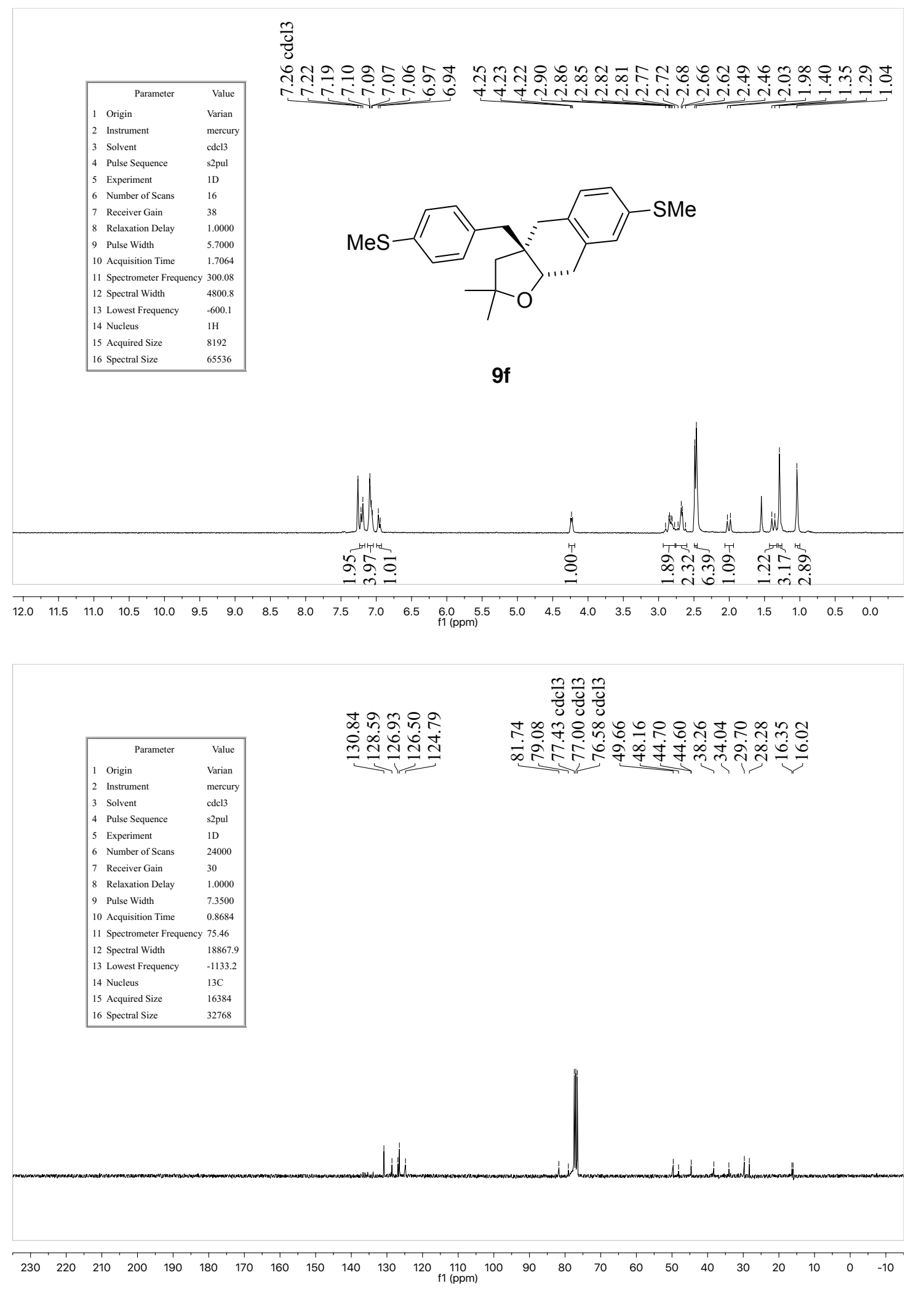



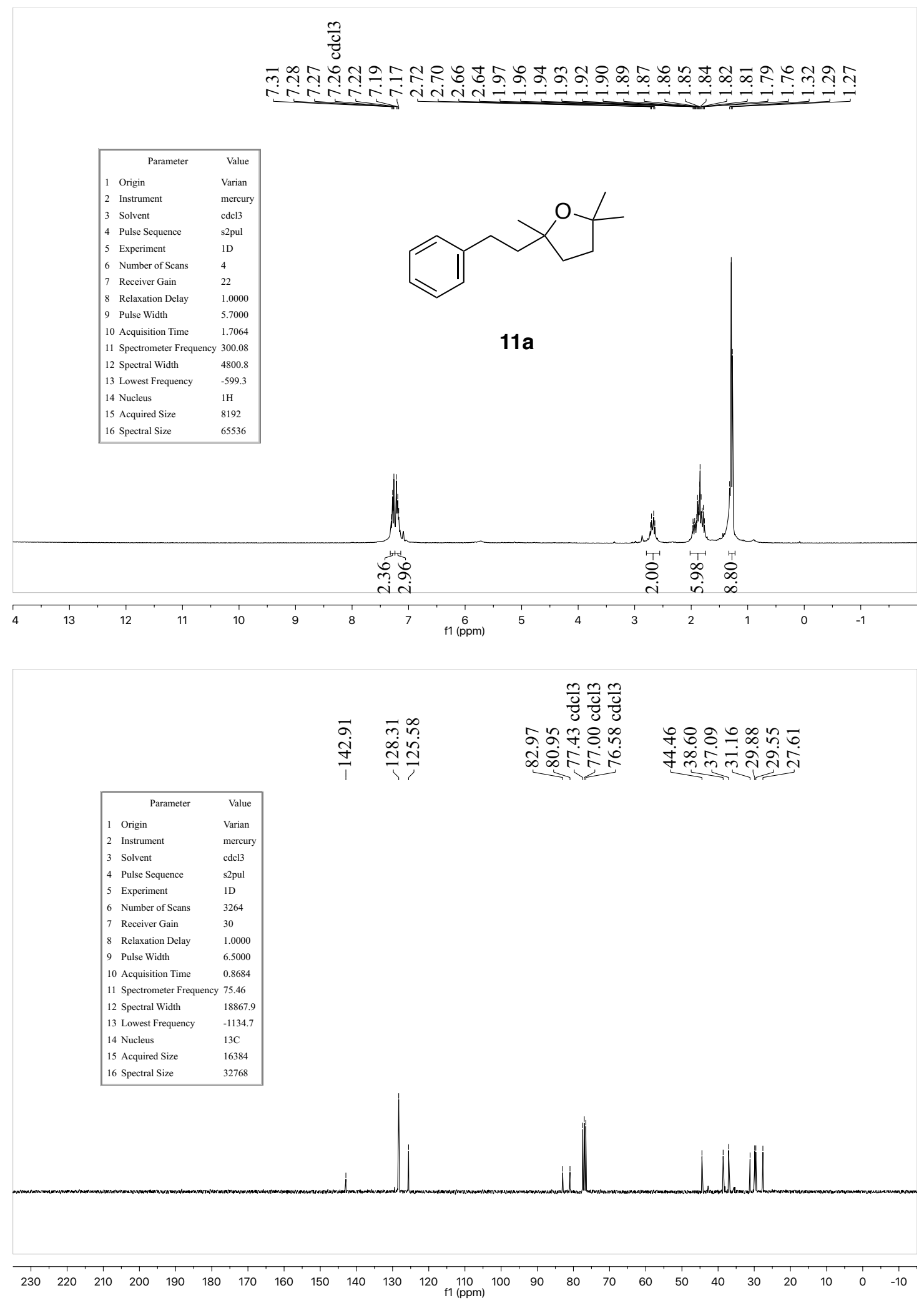